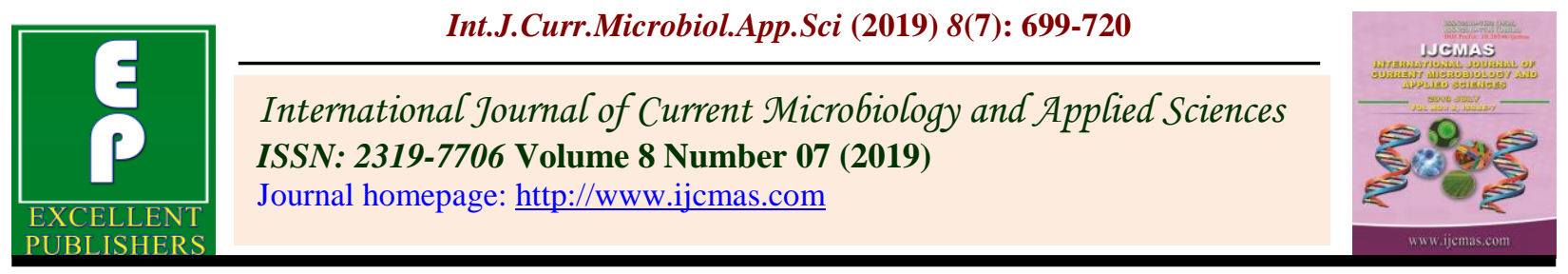

Original Research Article

https://doi.org/10.20546/ijcmas.2019.807.086

\title{
Correlation and Path Analysis Studies on Yield and its Components in Mung Bean [Vigna radiata (L.) Wilczek]
}

\author{
R.A. Jadhav*, S.P. Mehtre and D.K. Patil \\ Department of Agricultural Botany, College of Agriculture, Vasantrao Naik Marathwada \\ Krishi Vidyapeeth, Parbhani-431402 (Maharashtra), India \\ *Corresponding author
}

\begin{tabular}{|l|}
\hline $\begin{array}{l}\text { Ke y w or d s } \\
\text { Correlation, Mung } \\
\text { bean, Path analysis }\end{array}$ \\
\hline Article Info \\
\hline $\begin{array}{l}\text { Accepted: } \\
\text { 07 June } 2019 \\
\text { Available Online: } \\
\text { 10 July } 2019\end{array}$ \\
\hline \hline
\end{tabular}

A B S T R A C T

The correlation coefficients among 20 yield contributing traits with their path effects towards and seed yield using 70 genotypes of mung bean were investigated during Kharif 2015-16. The analysis of correlation coefficient suggested that the magnitude of genotypic correlations were higher than the corresponding phenotypic correlations. In present study, phenotypic and genotypic correlation of seed yield was positive and significant with days to $50 \%$ flowering, days to maturity, primary branches per plant, clusters per plant, pods per plant, pod length, 100-seed weight, shelling \%, biological yield per plant and harvest index. This suggests while selecting for improvement in seed yield these characters can be kept in mind provided the character should show high variability, which is basis for selection. However, seed yield per plant showed significant and negative association with protein content in both genotypic and phenotypic correlation studies. Hence, these traits also can be emphasized while selecting for improvement of seed yield. The path coefficient analysis revealed that biological yield per plant had highest direct effect on seed yield per plant followed by harvest index, days to maturity, number of clusters per plant, number of pods per plant, pod length, number of seeds per pod, number of pods per cluster, 100-seed weight both at genotypic and phenotypic level, whereas seed hardness, days to shattering and primary branches per plant at genotypic level only. This suggested that emphasis should be given to these traits in selection programme for improvement of seed yield in mung bean.

\section{Introduction}

Mung bean (Vigna radiata $\mathrm{L}$. Wilczek) is a third important pulse crop after Bengal gram and Red gram. It is mostly cultivated in countries like India, Pakistan, Bangladesh, Sri Lanka, Thailand, Laos, Cambodia, Vietnam, Indonesia, Malaysia, South China and Formosa. The area under mung bean in Maharashtra is 4.44 lakh ha with production of
2.60 lakh tonnes and productivity of 585 $\mathrm{Kg} / \mathrm{ha}$ during 2016-17. Maharashtra contributing $19.51 \%$ area with $30.92 \%$ contribution in production in the nation. The food values of mung bean lie in its high and easily digestible protein. Saleem et al., (1998) reported that seed contains components like, total protein (22.88-24.65\%), total amino acids (20.98-25.61\%), crude fibre (4.30-4.80\%) and lipids $(1.53-2.63 \%)$. 
Yield is a complex character and dependent on many component traits. Hence it is necessary to have knowledge on the extent of association between seed yield and yield contributing characters. Expression of various traits often varies with breeding materials and environments. Therefore, the information on character association between the traits themselves and with the yield for the specific breeding material is important for breeding high yielding genotypes. Correlation analysis provides the information of inter-relationship of important plant characters and hence, leads to a directional model for direct or indirect improvement in grain yield (Khan et al., 2004). Apart from the information on degree and direction of relationship between two or more traits, it is very important to know the cause of association by knowing the direct and indirect effects of independent characters on dependent character. The path analysis helps in partitioning the correlation coefficient of yield components with seed yield into its direct and indirect effects to ensure the actual contribution of an attribute as well as its influence through other traits. If the correlation is due to direct effect, it reflects true and perfect relationship and such characters can be selected for improving yield.

\section{Materials and Methods}

The material for present study comprised of 70 inter-specific derivatives of mung bean (including checks) collected from Plant Breeding Unit of Agricultural Research Station, Badnapur. Out of total 70 interspecific derivatives, 12 were derivatives of three way crosses $([(\mathrm{BM}-4 \times \mathrm{BWM}-29)] \times$ BM-2003-2), 12 were derivatives of four way crosses $([(B M-4 \times$ BWM-29) $\times$ BM-2003-2] $\times$ BM-2002-1), 41 were derivatives of single crosses (BM-4 × BWM-29) and five checks. So total of 70 derivatives were evaluated for 20 quantitative characters viz., days to $50 \%$ flowering, days to maturity, days to shattering, leaf length, leaf width, plant height, primary branches per plant, clusters per plant, pods per cluster, pods per plant, seeds per pod, pod length, 100-seed weight, shelling \%, biological yield per plant, harvest index, protein content, calcium content, seed hardness and seed yield per plant.

The present investigation was carried out during Kharif 2016 at experimental field of Agricultural Research Station, Badnapur. Sowing was done on $18^{\text {th }}$ June 2016. The randomized complete block design was followed with two replications and each treatment was in two rows with inter-row spacing of $45 \mathrm{~cm}$. All recommended package of practices were followed to raise a good crop. The phenotypic and genotypic correlation coefficients were worked out by using covariance technique as per Falconer (1964). Path coefficient analysis was carried out by Wright (1921) and illustrated by Dewey and Lu (1959).

\section{Results and Discussion}

The phenotypic and genotypic correlation coefficients between yield and yield component characters were estimated and presented in Table 1. The results were discussed character wise here as. Genotypic correlations in general are higher than phenotypic correlations indicating that the apparent associations are largely due to genetic reasons (Fig. 1 and 2).

Days to $50 \%$ flowering recorded highest significant positive association with days to shattering $\left(0.9937^{* *}\right.$ and $\left.0.9175^{* *}\right)$ followed by days to maturity $\left(0.9926^{* * *}\right.$ and $\left.0.9504^{* * *}\right)$, seed yield per plant $\left(0.3477^{* *}\right.$ and $\left.0.3192^{* *}\right), 100$ seed weight $\left(0.3417^{* *}\right.$ and $\left.0.2779^{*}\right)$, leaf width $\left(0.3190^{* * *}\right.$ and $\left.0.2798^{* * *}\right)$, plant height $\left(0.3117^{* *}\right.$ and $\left.0.2937^{* *}\right)$, pod length $\left(0.2474^{* *}\right.$ and $\left.0.1895^{*}\right)$ and biological yield per plant $\left(0.2334^{* *}\right.$ and $\left.0.2072^{*}\right)$ both at genotypic and 
phenotypic levels, respectively. This character also exhibited significant positive association with pods per plant $\left(0.1676^{*}\right)$ and significant negative association with protein content ($0.1877^{*}$ ) at genotypic level. This character also showed non-significant negative association with seed hardness $(-0.1032$ and $0.1026)$ followed by seeds per pod $(-0.0869$ and -0.0968$)$ and pods per cluster $(-0.0282$ and -0.0315) both at genotypic and phenotypic levels, respectively. These results are in conformity with the findings of Raturi et al., (2015), Kate et al., (2017), Lukman Hakim (2018) and Keerthiga et al., (2018) for days to $50 \%$ flowering.

Days to maturity showed highest significant positive association with days to shattering $\left(0.5836^{* *}\right.$ and $\left.0.9469^{* * *}\right)$ followed by plant height $\left(0.3535^{* *}\right.$ and $\left.0.3256^{* *}\right)$, seed yield per plant $\left(0.3391^{* *}\right.$ and $\left.0.3080^{* *}\right)$, leaf width $\left(0.3177^{* * *}\right.$ and $\left.0.2689^{* * *}\right), 100$-seed weight $\left(0.2979^{* *}\right.$ and $\left.0.2511^{* *}\right)$, pod length $\left(0.2445^{* *}\right.$ and $0.2294^{* * *}$ ) and biological yield per plant $\left(0.2078^{* *}\right.$ and $\left.0.1859^{*}\right)$ both at genotypic and phenotypic levels, respectively. This character also exhibited significant positive association with pods per plant $\left(0.168^{*}\right)$ and negative association with protein content $\left(-0.1877^{*}\right)$ at genotypic level (Table 1). This character also showed non-significant negative association with seed hardness (-0.1122 and -0.1217), protein content $(-0.1203$ and -0.1034$)$ and seeds per pod $(-0.01619$ and -0.0547$)$ both at genotypic and phenotypic levels, respectively. Similar results are in line with the earlier findings of Kate et al., (2017), Lukman Hakim (2018) and Keerthiga et al., (2018) for days to maturity.

Days to shattering showed highest significant positive association with leaf width $\left(0.3722^{* *}\right.$ and $0.2509^{* *}$ ) followed by seed yield per plant $\left(0.3431^{* *}\right.$ and $\left.0.3135^{* * *}\right), 100$-seed weight $\left(0.3071^{* *}\right.$ and $\left.0.2281^{* *}\right)$, plant height $\left(0.2971^{* *}\right.$ and $\left.0.2633^{* *}\right)$, biological yield per plant $\left(0.2262^{* *}\right.$ and $\left.0.2209^{* *}\right)$ and pods per plant $\left(0.2071^{* *}\right.$ and $\left.0.1822^{*}\right)$ both at genotypic and phenotypic level, respectively. This character also exhibited significant positive association with primary branches per plant $\left(0.1970^{*}\right)$ and pod length $\left(0.1708^{*}\right)$ at genotypic and phenotypic levels, respectively. (Table 4.7) Non-significant negative association with seed hardness $(-0.1154$ and -0.1185$)$ followed by protein content $(-0.1132$ and -0.0961$)$, seeds per pod $(-0.0414$ and -0.0312$)$ and calcium content $(-0.0315$ and -0.0247$)$ both at genotypic and phenotypic levels, respectively.

The character plant height showed highly significant positive association with leaf width $\left(0.5617^{* *}\right.$ and $\left.0.4796^{* *}\right)$ followed by pod length $\left(0.4241^{* *}\right.$ and $\left.0.3235^{* *}\right)$, leaf length $\left(0.1971^{*}\right.$ and $\left.0.1684^{*}\right)$ and harvest index $\left(0.1802^{*}\right.$ and $\left.0.1679^{*}\right)$. This character also exhibited non-significant negative association with clusters per plant $(-0.1395$ and -0.1365$)$ followed by seed hardness $(-0.1341$ and $0.1273)$ and pods per plant (-0.0132 and $0.0119)$ both at genotypic and phenotypic levels, respectively. Number of primary branches per plant had showed significant positive association with clusters per plant $\left(0.5617^{* *}\right.$ and $\left.0.2923^{* *}\right)$ followed by 100 -seed weight $\left(0.4241^{* *}\right.$ and $\left.0.2039^{*}\right)$ and seed yield per plant $\left(0.2428^{* *}\right.$ and $\left.0.2242^{* *}\right)$ were observed. Again it showed positive significant association with leaf width $\left(0.1972^{*}\right)$ and protein content $\left(0.1802^{*}\right)$ at genotypic level and pods per plant $\left(0.2815^{* *}\right)$, seeds per pod $\left(0.2481^{* *}\right)$, biological yield per plant $\left(0.2148^{*}\right)$ and leaf length $\left(0.1743^{*}\right)$ at phenotypic level. Kritika and Yadav (2017), Kate et al., (2017), Ghimire et al., (2017) and Keerthiga et al., (2018) also obtained similar results for primary branches per plant. Again this character showed non-significant negative association with pods per cluster (-0.1395) and seeds per pod (-0.0132) at genotypic levels and protein content (-0.0266) and calcium content (-0.0860) at phenotypic level. 
The trait leaf length showed significant positive association with leaf width $\left(0.6979^{* *}\right.$ and $0.4766^{* * *}$ ) followed by pod length $\left(0.3418^{* *}\right.$ and $\left.0.2411^{* *}\right)$ and pods per plant $\left(0.3253^{* *}\right.$ and $\left.0.2539^{* *}\right)$ both at genotypic and phenotypic levels was observed. This character showed significant negative correlation with clusters per plant $\left(-0.2438^{* *}\right.$ and $-0.1761^{*}$ ) both at genotypic and phenotypic levels respectively. Again this trait showed significant positive association with 100-seed weight $\left(0.2289^{* * *}\right)$ followed by seed hardness $\left(0.2208^{* *}\right)$ and calcium content $\left(0.1763^{*}\right)$ and significant negative association with biological yield per plant $\left(-0.1884^{*}\right)$ at genotypic level. The character leaf length showed non-significant negative association with seed yield per plant (-0.1289 and $0.0991)$ followed by protein content $(-0.0736$ and -0.0658$)$ and shelling \% (-0.0603 and 0.0684 ) both at genotypic and phenotypic levels, respectively.

The character leaf width showed highly significant positive association with pod length $\left(0.4145^{* *}\right.$ and $\left.0.2254^{* *}\right)$ followed by 100 -seed weight $\left(0.3077^{* *}\right.$ and $\left.0.2669^{* * *}\right)$ both at phenotypic and genotypic levels. Harvest index showed significant positive association $\left(0.1860^{*}\right)$ with this trait at genotypic level. It also showed non-significant negative association with protein content $(-0.0525$ and $0.0174)$, clusters per plant $(-0.0497$ and $0.0434)$, shelling \% (-0.0494 and -0.0299), biological yield per plant $(-0.0288$ and $0.0648)$ and seed hardness (-0.0266 and 0.0107 ) both at genotypic and phenotypic levels, respectively.

The character clusters per plant showed significant positive association with pods per plant $\left(0.5946^{* *}\right.$ and $\left.0.5775^{* * *}\right)$ followed by seed yield per plant $\left(0.3787^{* *}\right.$ and $\left.0.3556^{* *}\right)$, biological yield per plant $\left(0.3379^{* *}\right.$ and $\left.0.3166^{* *}\right), 100$-seed weight $\left(0.2261^{* *}\right.$ and $\left.0.1926^{*}\right)$ and shelling \% $\left(0.2053^{*}\right.$ and $\left.0.1823^{*}\right)$. This character showed nonsignificant negative association with calcium content $(-0.1022$ and -0.1016$)$ followed by protein content $(-0.0995$ and -0.0880$)$, seed hardness (-0.0833 and -0.0902) and pod length $(-0.0102$ and -0.0123$)$ both at genotypic and phenotypic levels respectively. Similar results were recorded by Baisakh et al., (2015), Hemavathy et al., (2015), Keerthiga et al., (2018) and Divya Ramakrishnan et al., (2018) for the trait clusters per plant.

Trait pods per cluster had showed significant positive association with pods per plant $\left(0.3131^{* * *}\right.$ and $\left.0.2883^{* *}\right)$ followed by harvest index $\left(0.2911^{* * *}\right.$ and $\left.0.2608^{* * *}\right)$ and seed hardness $\left(0.2400^{* * *}\right.$ and $\left.0.2075^{* *}\right)$ both at genotypic and phenotypic levels, respectively. This character also showed significant positive association with shelling $\%\left(0.1878^{*}\right)$ at genotypic level. Non-significant negative association with pod length $(-0.1325$ and $0.0981)$, seeds per pod $(-0.1225$ and -0.1147$)$ and protein content $(-0.0209$ and -0.0131$)$ both at genotypic and phenotypic levels, respectively. It also showed non-significant positive association with seed yield per plant (0.0838 and 0.0903$)$ followed by calcium content $(0.0542$ and 0.0470$)$ and 100 -seed weight $(0.0201$ and 0.0164$)$ both at genotypic and phenotypic levels, respectively.

Pods per plant showed significant positive association with seed yield per plant $\left(0.3178^{* *}\right.$ and $0.3084^{* *}$ ) followed by biological yield per plant $\left(0.2607^{* *}\right.$ and $\left.0.2422^{* *}\right)$, harvest index $\left(0.2205^{* *}\right.$ and $\left.0.2106^{* *}\right)$ and 100 -seed weight $\left(0.1952^{*}\right.$ and $\left.0.1647^{*}\right)$. It also showed significant negative association with protein content $\left(-0.2219^{* *}\right.$ and $\left.-0.2093^{*}\right)$ both at genotypic and phenotypic levels, respectively. Similar results were obtained by Hemavathy et al., (2015), Kritika and Yadav (2017), Keerthiga et al., (2018), Lukman Hakim (2018) and Divya Ramakrishnan et al., (2018) for pods per plant. Pods per plant showed non- 
significant positive association with seed hardness (0.0929 and 0.0181) followed by shelling \% (0.0832 and 0.0825), pod length (0.0730 and 0.0569), seeds per pod (0.0711 and 0.0747$)$ and calcium content (0.0171 and $0.0181)$.

Seeds per pod had showed significant positive association with pod length $\left(0.3065^{* *}\right.$ and $0.2518^{* * *}$ ). Also it showed negative association with calcium content $\left(-0.1860^{*}\right.$ and $\left.-0.1765^{*}\right)$ both at genotypic and phenotypic levels, respectively. Significant positive association with protein content $\left(0.1778^{*}\right)$ at genotypic level. Again it showed non-significant negative association with harvest index (0.0198 and -0.0030$)$ both at genotypic and phenotypic levels, respectively. Seeds per pod showed non-significant positive association was seed yield per plant $(0.1008$ and 0.1040$)$ followed by shelling \% (0.0901 and 0.0849), biological yield per plant (0.0407 and 0.0397) and 100-seed weight (0.0133 and 0.0171).

Pod length showed significant positive association with 100 -seed weight $\left(0.5032^{* *}\right.$ and $0.3916^{* * *}$ ) followed by seed yield per plant $\left(0.3049^{* *}\right.$ and $\left.0.2537^{* *}\right)$, shelling \% $\left(0.2395^{* *}\right.$ and $0.1820^{* * *}$ ) and biological yield per plant $\left(0.1981^{*}\right.$ and $\left.0.1681^{*}\right)$ both at genotypic and phenotypic levels respectively. Nonsignificant negative association with protein content (-0.0613 and -0.0369) and seed hardness $(-0.0559$ and -0.0252$)$ both at genotypic and phenotypic levels, respectively. The results are in agreement with Ahmad et al., (2013), Baisakh et al., (2015) and Das and Barua (2015) and non-significant positive association with harvest index (0.1398 and $0.1008)$ and calcium content $(0.1036$ and $0.0824)$ both at genotypic and phenotypic levels, respectively.

100-seed weight showed significant positive association with seed yield $\left(0.4248^{* *}\right.$ and $0.3890^{* *}$ ) followed by biological yield per plant $\left(0.3806^{* *}\right.$ and $\left.0.3077^{* *}\right)$, shelling \% $\left(0.2603^{* *}\right.$ and $\left.0.2526^{* *}\right)$ and harvest index $\left(0.2078^{* *}\right.$ and $\left.0.1847^{*}\right)$. It also showed nonsignificant negative association with calcium content $(-0.1112$ and -0.0931$)$ and protein content $(-0.1031$ and -0.0431$)$ both at genotypic and phenotypic levels respectively. Ahmad et al., (2013), Hemavathy et al., (2015), Das and Barua (2015), Kritika and Yadav (2017), Kate et al., (2017), Ghimire et al., (2017) and Keerthiga et al., (2018) found similar results for 100 -seed weight. and nonsignificant positive association with seed hardness $(0.0531$ and 0.0579$)$ both at genotypic and phenotypic levels, respectively.

The character shelling $\%$ had showed significant positive association with seed yield per plant $\left(0.5165^{* *}\right.$ and $\left.0.4929^{* *}\right)$ followed by biological yield per plant $\left(0.4130^{* *}\right.$ and $\left.0.3722^{* *}\right)$ and harvest index $\left(0.2753^{* *}\right.$ and $\left.0.2583^{* *}\right)$. Again it showed significant negative association with calcium content ($0.3885^{* *}$ and $-0.3524^{* *}$ ) both at genotypic and phenotypic levels respectively. It also showed non-significant negative association with protein content (-0.15662 and -0.1109) both at genotypic and phenotypic levels respectively and seed hardness (-0.0110) at genotypic level. Muralidhara et al., (2015) also obtained similar results. Also non-significant positive association was showed with seed hardness (0.0103) at phenotypic level only.

The trait biological yield per plant had showed significant positive association with seed yield per plant $\left(0.8113^{* *}\right.$ and $\left.0.7497^{* *}\right)$ both at genotypic and phenotypic levels respectively. Again it showed significant negative association with calcium content $\left(-0.1982^{*}\right)$ at phenotypic level. Character showed nonsignificant negative association with seed hardness $(-0.1085$ and -0.1005$)$ followed by protein content $(-0.1027$ and -0.0926$)$ and harvest index $(-0.0268$ and -0.0440$)$ both at genotypic and phenotypic levels respectively 
and calcium content (-0.2104) at genotypic level. The results are in agreement with the findings of Narasimhulu et al., (2013), Canci and Toker (2014), Kritika and Yadav (2017) and Ghimire et al., (2017).

Harvest index has showed significant positive association with seed yield per plant $\left(0.5009^{* *}\right.$ and $0.4809^{* * *}$ ) followed by seed hardness $\left(0.2020^{*}\right.$ and $\left.0.2026^{*}\right)$ and significant negative association with protein content $\left(-0.1905^{*}\right.$ and $\left.-0.1681^{*}\right)$ both at genotypic and phenotypic levels respectively. The findings are similar with the conclusions of Pandey et al., (2007), Gul et al., (2008), Rozina et al., (2008) and Tejbir et al., (2009) for harvest index and nonsignificant positive association with calcium content $(0.0977$ and 0.0938$)$ both at genotypic and phenotypic levels, respectively.

The character protein content showed significant negative association with seed yield per plant $\left(-0.2237^{*}\right.$ and $\left.-0.1909^{*}\right)$ both at genotypic and phenotypic levels respectively. Again it showed non-significant negative association with seed hardness (-0.0892 and 0.0893 ) both at genotypic and phenotypic levels respectively and non-significant positive association with calcium content (0.0856 and 0.0766) both at genotypic and phenotypic levels, respectively.

The trait calcium content showed nonsignificant negative association with seed yield per plant $(-0.1378$ and -0.1344$)$ followed by seed hardness $(-0.0919$ and -0.0906$)$ both at genotypic and phenotypic levels respectively. The character seed hardness showed non-significant negative association with seed yield per plant $(-0.0086$ and -0.001$)$ both at genotypic and phenotypic levels respectively.

This suggests while selecting for improvement in seed yield these characters can be kept in mind provided the character should show high variability, which is basis for selection. However, seed yield per plant showed significant and negative association with protein content in both genotypic and phenotypic correlation studies. Hence, these traits also can be emphasized while selecting for improvement of seed yield. In present study, phenotypic and genotypic correlation of seed yield was positive and significant with days to $50 \%$ flowering, days to maturity, days to shattering, primary branches per plant, clusters per plant, pods per plant, pod length, 100 -seed weight, shelling \%, biological yield per plant and harvest index.

\section{Path coefficient analysis}

The direct and indirect effects of yield contributing traits have given in Figure 3 and 4.

\section{Days to $50 \%$ flowering}

At genotypic level, this trait had positive significant association $\left(0.3477^{* *}\right)$ and negative direct effect $(-0.2726)$ on seed yield per plant. It also had positive indirect effects via., days to maturity (0.3327), biological yield per plant (0.1841), harvest index (0.0716), protein content $(0.0260)$, pod length $(0.0195)$, days to shattering (0.0139), clusters per plant (0.0080), pods per cluster (0.0012), primary branches per plant (0.0005), calcium content (0.0002) and 100-seed weight (0.0039) at genotypic level.

This trait had significant positive correlation $\left(0.3192^{* *}\right)$ and negative direct effect $(-0.0816)$ on seed yield per plant at phenotypic level. It also expressed positive indirect effects via., biological yield per plant (0.1412), days to maturity (0.1134), harvest index (0.0554), pod length (0.0066), 100-seed weight (0.0064) and protein content $(0.0060)$ and negative indirect effects via., days to shattering (-0.0825), plant height (-0.0038), leaf length (-0.0021), 
primary branches per plant $(-0.0014)$, seeds per pod $(-0.0084)$, pods per plant $(-0.0031)$, pods per cluster $(-0.0001)$, calcium content $(-$ $0.0001)$ and seed hardness (-0.0008).

The direct effects of plant height are negative and the correlation coefficients are significantly positive at both phenotypic and genotypic level. Bastian et al., (2001), Saifullah et al., (2002), Madrap et al., (2003) and Kumar and Katiyar (2015) found same results for days to $50 \%$ flowering.

\section{Days to maturity}

At genotypic level, this character showed significant positive association $\left(0.3391^{* *}\right)$ and positive direct effect $(0.3425)$ on seed yield per plant. It also possessed positive indirect effects via., biological yield per plant (0.1638), harvest index (0.0796), protein content (0.0167), pod length (0.0193), days to shattering (0.0140), clusters per plant (0.0059), 100-seed weight (0.0034), primary branches per plant (0.0004) and negative indirect effects via., days to $50 \%$ flowering ($0.2633)$, plant height $(-0.0166)$, leaf length ($0.0029)$, leaf width $(-0.0016)$, pods per cluster $(-0.0002)$, pods per plant $(-0.0157)$, seeds per pod (-0.0031), shelling \% (-0.003), calcium content (-0.0004) and seed hardness (-0.0024).

The character expressed significant positive association $\left(0.3080^{* *}\right)$ and positive direct effect (0.1193) on seed yield per plant at phenotypic level. It also had positive indirect effects via., days to $50 \%$ flowering (0.0775), leaf width (0.0057), clusters per plant (0.0035), pod length (0.0080), 100-seed weight (0.0058), shelling \% (0.0016), biological yield per plant (0.1267), harvest index (0.0582), protein content (0.0039), calcium content (0.0004), while it showed negative indirect effects via., days to shattering (-0.0851), plant height (-0.0043), primary branches per plant (0-.0012), leaf length $(-0.0031)$, pods per plant $(-0.0034)$, seeds per pod (-0.0047) and seed hardness ($0.0009)$. Days to maturity is in accordance with the results of Kumawat and Raje (2005), Reddy et al., (2011), Prasanna et al., (2013) and Kumar and Katiyar (2015).

\section{Days to shattering}

At genotypic level, this trait showed positive significant association $\left(0.3431^{* * *}\right)$ and positive direct effect (0.0140) on seed yield per plant. It also exhibited positive indirect effects via., days to maturity (0.3428), biological yield per plant $(0.1784)$, harvest index $(0.0715)$, protein content (0.0157), 100-seed weight (0.0038), pod length (0.0097), clusters per plant (0.0133), primary branches per plant (0.0007), calcium content (0.007) and negative indirect effects via., days to $50 \%$ flowering (-0.2647), plant height $(-0.0140)$, leaf length $(-0.0021)$, leaf width (-0.0018), pods per cluster ($0.0006)$, pods per plant $(-0.0194)$, seeds per pod $(-0.0021)$, shelling \% (-0.0002) and seed hardness $(-0.0025)$.

The character expressed significant positive association $\left(0.3135^{* *}\right)$ and negative direct effect (-0.0899) on seed yield per plant at phenotypic level. It also had positive indirect effects via., days to maturity (0.1129), days to $50 \%$ flowering (0.0748), leaf width (0.0054), clusters per plant $(0.0084)$, pods per cluster (0.0001), pod length (0.0059), 100-seed weight (0.0053), biological yield per plant (0.1505), harvest index (0.0535), protein content (0.0036), while it showed negative indirect effects via., plant height (-0.0034), primary branches per plant (-0.0022), shelling $\%$ (-0.0001), calcium content (-0.0004) and seed hardness (-0.0009). Thus this character showed positive direct effects coupled with highly significant positive correlation on seed yield per plant at genotypic level and showed negative direct effect with highly significant positive association at phenotypic level (Table 2). 
Fig.1 Genotypic correlations of 20 characters on seed yield in Mung bean

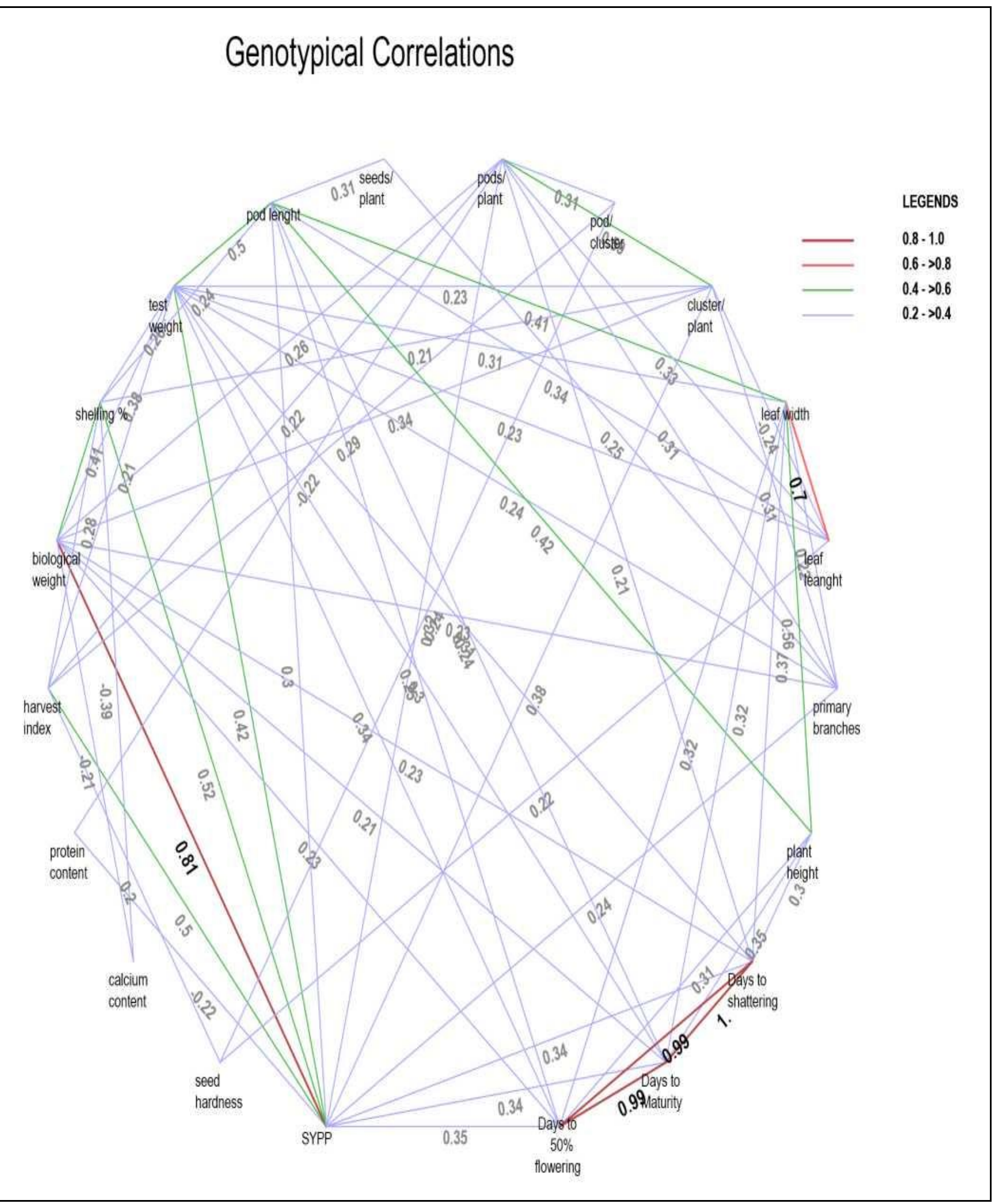


Fig.2 Phenotypic correlations of 20 characters on seed yield in Mung bean

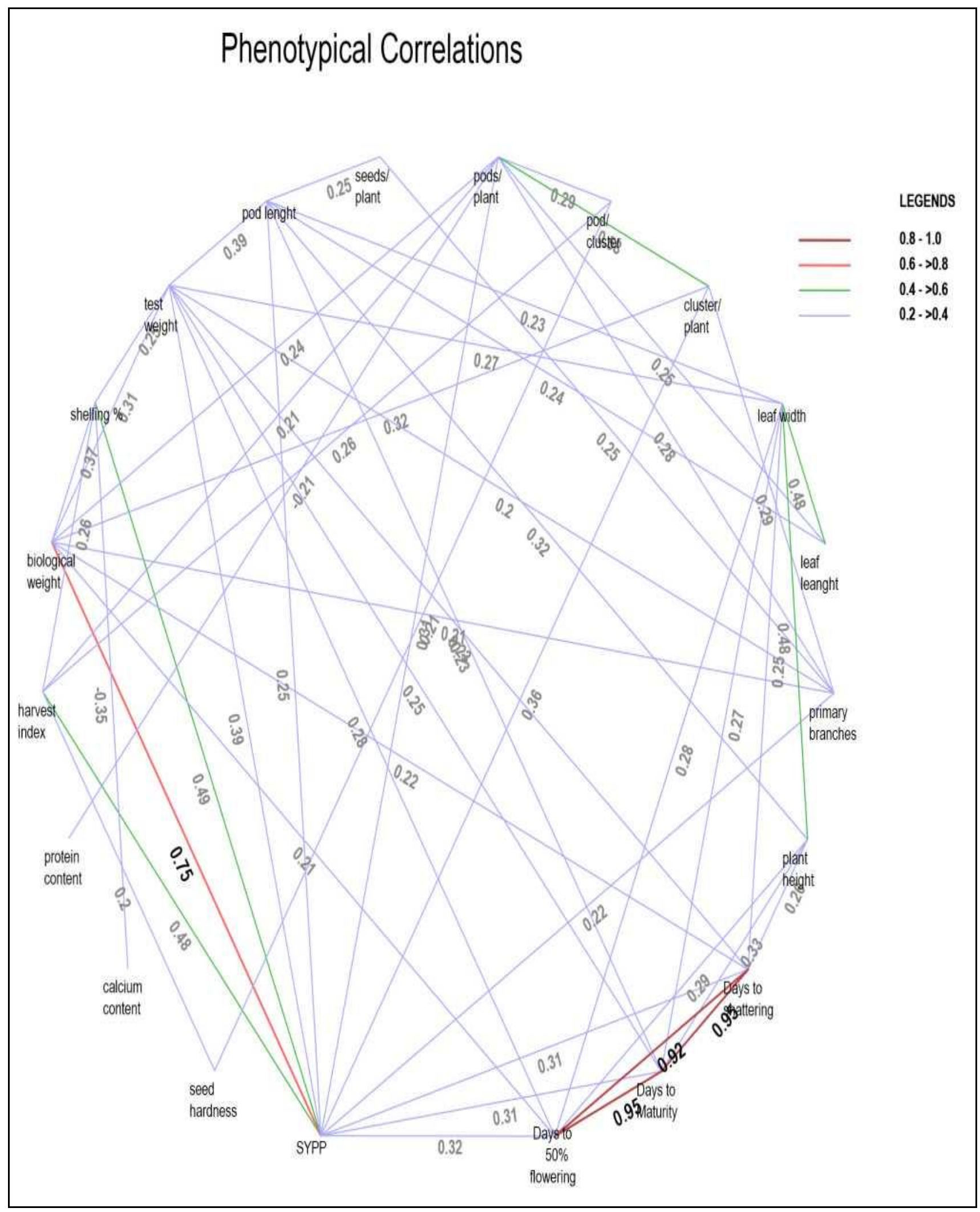


Fig.3 Genotypic path diagram showing direct and indirect effects of yield components on seed yield per plant in Mung bean

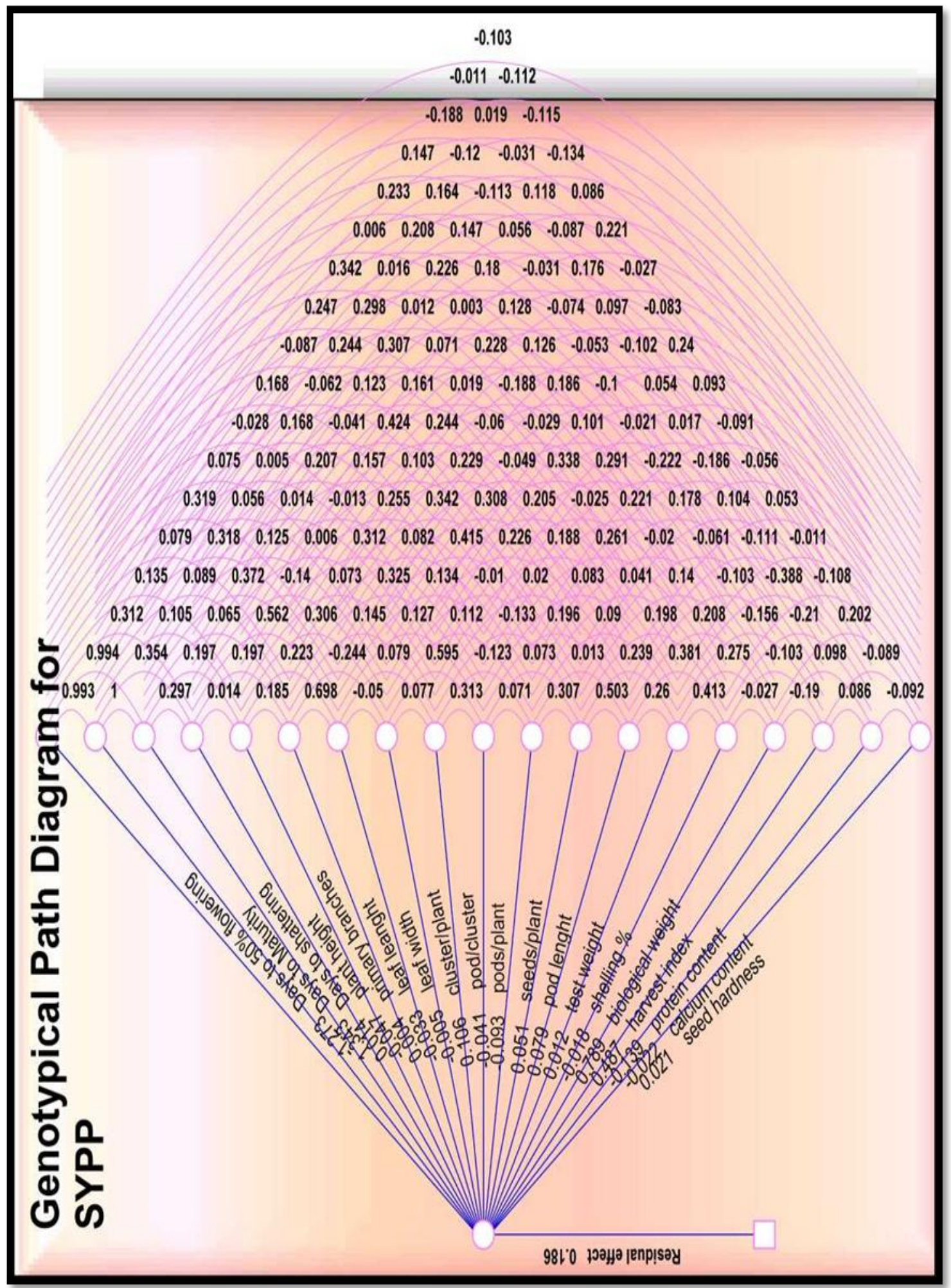

(SYPP-Seed Yield Per Plant) 
Fig.4 Phenotypic path diagram showing direct and indirect effects of yield components on seed yield per plant in Mung bean

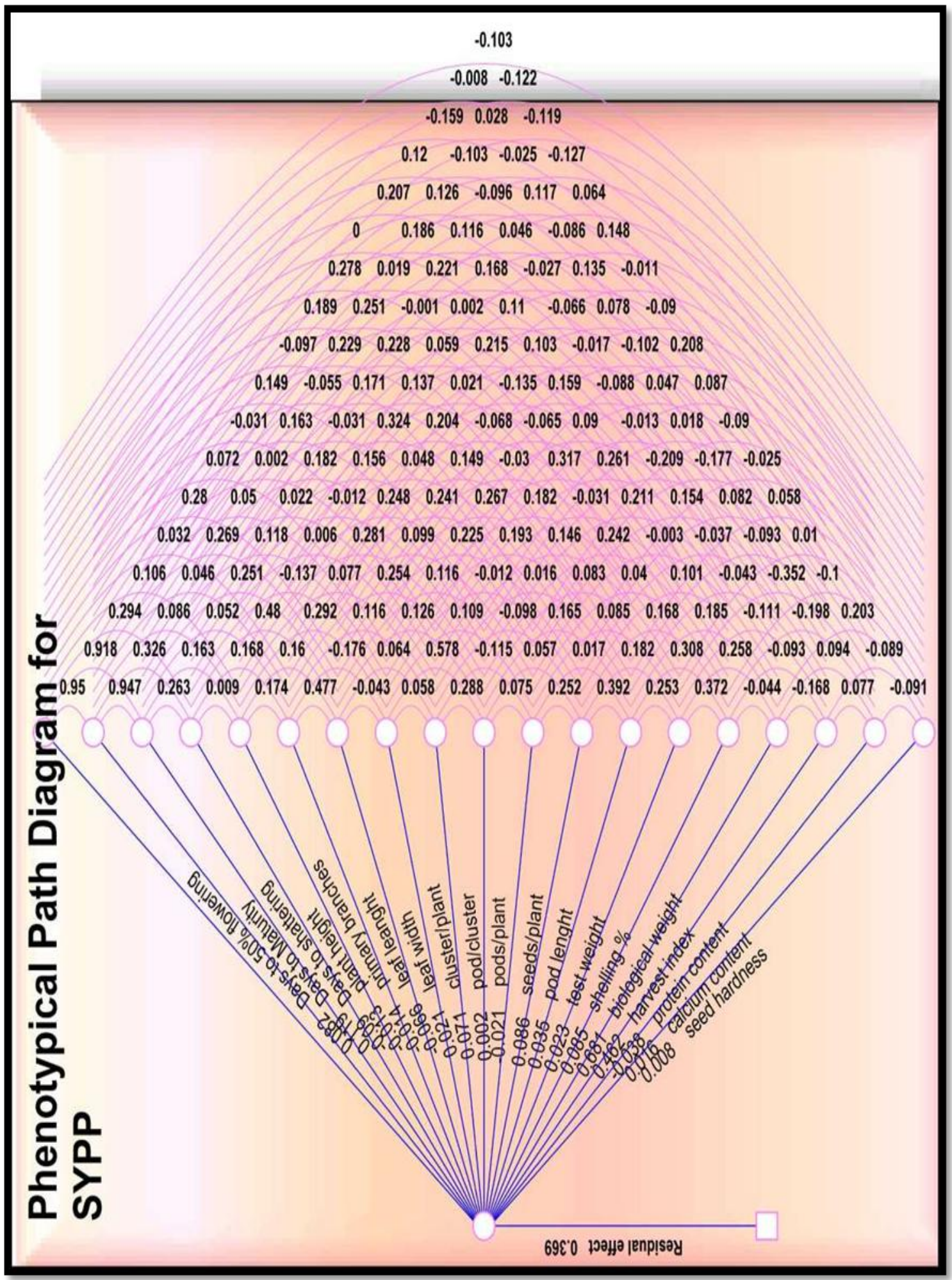

(SYPP-Seed Yield Per Plant) 
Table.1 Genotypic and phenotypic correlation co-efficient between different traits mung bean

\begin{tabular}{|c|c|c|c|c|c|c|c|c|c|c|c|c|c|c|c|c|c|c|c|c|c|}
\hline Character & $\begin{array}{c}\text { Correlatio } \\
n\end{array}$ & $\begin{array}{c}\text { Days to } \\
\mathbf{5 0 \%} \\
\text { flowering }\end{array}$ & $\begin{array}{c}\text { Days to } \\
\text { maturi } \\
\text { ty }\end{array}$ & $\begin{array}{c}\begin{array}{c}\text { Days to } \\
\text { shattering }\end{array} \\
\text { s. }\end{array}$ & $\begin{array}{c}\begin{array}{c}\text { Plant } \\
\text { height }\end{array}\end{array}$ & $\begin{array}{c}\text { Primary } \\
\text { branches/ } \\
\text { plant }\end{array}$ & $\begin{array}{c}\text { Leaf } \\
\text { length }\end{array}$ & $\begin{array}{c}\begin{array}{c}\text { Leaf } \\
\text { width }\end{array} \\
\end{array}$ & $\begin{array}{c}\text { Clusters/ } \\
\text { plant }\end{array}$ & $\begin{array}{l}\text { Pods/ } \\
\text { cluster }\end{array}$ & $\begin{array}{c}\text { Pods/pla } \\
\text { nt }\end{array}$ & $\begin{array}{c}\text { Seeds/po } \\
\text { d }\end{array}$ & $\begin{array}{c}\text { Pod } \\
\text { length }\end{array}$ & $\begin{array}{c}\text { 100-Seed } \\
\text { weight }\end{array}$ & $\begin{array}{l}\text { Shellin } \\
\text { g \% }\end{array}$ & $\begin{array}{c}\text { Biologica } \\
\text { 1 } \\
\text { yield/pla } \\
\text { nt }\end{array}$ & $\begin{array}{c}\text { Harvest } \\
\text { index }\end{array}$ & $\begin{array}{l}\text { Protein } \\
\text { content }\end{array}$ & $\begin{array}{l}\text { Calcium } \\
\text { content }\end{array}$ & $\begin{array}{c}\text { Seed } \\
\text { hardnes } \\
\mathrm{s}\end{array}$ & $\begin{array}{c}\text { Seed } \\
\text { yield/pla } \\
\text { nt }\end{array}$ \\
\hline $\begin{array}{l}\text { Days to } \\
50 \% \\
\text { flowering }\end{array}$ & $\begin{array}{l}\mathbf{G} \\
\mathbf{P}\end{array}$ & 1 & $\begin{array}{l}0.9926 * \\
* \\
0.9504 * \\
*\end{array}$ & $\begin{array}{l}0.9937^{* *} \\
0.9175^{* * *}\end{array}$ & $\begin{array}{l}0.3117^{* * *} \\
0.2937^{* * *}\end{array}$ & $\begin{array}{l}0.1352 \\
0.1057\end{array}$ & $\begin{array}{l}0.0790 \\
0.0318\end{array}$ & $\begin{array}{l}0.3190^{* *} \\
0.2798 * *\end{array}$ & $\begin{array}{l}0.0748 \\
0.0722\end{array}$ & $\begin{array}{l}-0.0282 \\
-0.0315\end{array}$ & $\begin{array}{l}0.1676 \\
0.1487\end{array}$ & $\begin{array}{l}-0.0869 \\
-0.0968\end{array}$ & $\begin{array}{l}0.2474 * * \\
0.1895 *\end{array}$ & $\begin{array}{l}0.3417 * * \\
0.2779 *\end{array}$ & $\begin{array}{c}0.0063 \\
-0.0004\end{array}$ & $\begin{array}{c}0.2334^{* * *} \\
0.2072^{*}\end{array}$ & $\begin{array}{l}0.1471 \\
0.1199\end{array}$ & $\begin{array}{c}-0.1877^{*} \\
-0.1591\end{array}$ & $\begin{array}{l}-0.0108 \\
-0.0080\end{array}$ & $\begin{array}{l}-0.1032 \\
-0.1026\end{array}$ & $\begin{array}{l}0.3477^{* * *} \\
0.3192^{* * *}\end{array}$ \\
\hline $\begin{array}{l}\text { Days to } \\
\text { maturity }\end{array}$ & $\begin{array}{l}\mathbf{G} \\
\mathbf{P}\end{array}$ & & 1 & $\begin{array}{l}0.5836^{* * *} \\
0.9469^{* *}\end{array}$ & $\begin{array}{l}0.3535 * * \\
0.3256 * *\end{array}$ & $\begin{array}{l}0.1054 \\
0.0856\end{array}$ & $\begin{array}{l}0.0890 \\
0.0462\end{array}$ & $\begin{array}{l}0.3177 * * \\
0.2689 * *\end{array}$ & $\begin{array}{l}0.0558 \\
0.0499\end{array}$ & $\begin{array}{l}0.0047 \\
0.0024\end{array}$ & $\begin{array}{l}0.1680^{*} \\
0.1634\end{array}$ & $\begin{array}{l}-0.0619 \\
-0.0547\end{array}$ & $\begin{array}{l}0.2445^{* *} \\
0.2294^{* *}\end{array}$ & $\begin{array}{l}0.2979 \text { ** } \\
0.2511^{* *}\end{array}$ & $\begin{array}{l}0.0160 \\
0.0191\end{array}$ & $\begin{array}{l}0.2078^{* * *} \\
0.1859^{*}\end{array}$ & $\begin{array}{l}0.1635 \\
0.1259\end{array}$ & $\begin{array}{l}-0.1203 \\
-0.1034\end{array}$ & $\begin{array}{l}0.0191 \\
0.0276\end{array}$ & $\begin{array}{l}-0.1122 \\
-0.1217\end{array}$ & $\begin{array}{l}0.3391 * * \\
0.3080 * *\end{array}$ \\
\hline $\begin{array}{l}\text { Days to } \\
\text { shattering }\end{array}$ & $\begin{array}{l}\text { G } \\
\mathbf{P}\end{array}$ & & & 1 & $\begin{array}{l}0.2971 \text { ** } \\
0.2633^{* * *}\end{array}$ & $\begin{array}{c}0.1970^{*} \\
0.1629\end{array}$ & $\begin{array}{l}0.0648 \\
0.0518\end{array}$ & $\begin{array}{l}0.3722^{* * *} \\
0.2509^{* *}\end{array}$ & $\begin{array}{l}0.1255 \\
0.1185\end{array}$ & $\begin{array}{l}0.0144 \\
0.0217\end{array}$ & $\begin{array}{l}0.2071^{* *} \\
0.1822^{*}\end{array}$ & $\begin{array}{l}-0.0414 \\
-0.0312\end{array}$ & $\begin{array}{c}0.1231 \\
0.1708^{*}\end{array}$ & $\begin{array}{l}0.3071^{* * *} \\
0.2281^{* *}\end{array}$ & $\begin{array}{c}0.0120 \\
-0.0010\end{array}$ & $\begin{array}{l}0.2262 * * \\
0.2209 * *\end{array}$ & $\begin{array}{l}0.1470 \\
0.1158\end{array}$ & $\begin{array}{l}-0.1132 \\
-0.0961\end{array}$ & $\begin{array}{l}-0.0315 \\
-0.0247\end{array}$ & $\begin{array}{l}-0.1154 \\
-0.1185\end{array}$ & $\begin{array}{l}-0.1431 \\
-0.1135\end{array}$ \\
\hline $\begin{array}{l}\text { Plant } \\
\text { height }\end{array}$ & $\begin{array}{l}\mathbf{G} \\
\mathbf{P}\end{array}$ & & & & 1 & $\begin{array}{l}0.0137 \\
0.0088\end{array}$ & $\begin{array}{l}0.1971^{*} \\
0.1684^{*}\end{array}$ & $\begin{array}{l}0.5617 * * \\
0.4796 * *\end{array}$ & $\begin{array}{l}-0.1395 \\
-0.1365\end{array}$ & $\begin{array}{l}0.0056 \\
0.0056\end{array}$ & $\begin{array}{l}-0.0132 \\
-0.0119\end{array}$ & $\begin{array}{l}0.1566 \\
0.1565\end{array}$ & $\begin{array}{l}0.4241^{* * *} \\
0.3235^{* * *}\end{array}$ & $\begin{array}{l}0.1607 \\
0.1372\end{array}$ & $\begin{array}{l}0.0705 \\
0.0589\end{array}$ & $\begin{array}{l}0.0026 \\
0.0021\end{array}$ & $\begin{array}{l}0.1802^{*} \\
0.1679^{*}\end{array}$ & $\begin{array}{l}0.0562 \\
0.0456\end{array}$ & $\begin{array}{l}0.1183 \\
0.1166\end{array}$ & $\begin{array}{l}-0.1341 \\
-0.1273\end{array}$ & $\begin{array}{l}0.1304 \\
0.1268\end{array}$ \\
\hline $\begin{array}{l}\text { Primary } \\
\text { branches/ } \\
\text { plant }\end{array}$ & $\begin{array}{l}\mathbf{G} \\
\mathbf{P}\end{array}$ & & & & & 1 & $\begin{array}{c}0.0137 \\
0.1743^{*}\end{array}$ & $\begin{array}{c}0.1971 * \\
0.1598\end{array}$ & $\begin{array}{l}0.5617^{* *} \\
0.2923^{* *}\end{array}$ & $\begin{array}{l}-0.1395 \\
0.0766\end{array}$ & $\begin{array}{c}0.0056 \\
0.2815^{* * *}\end{array}$ & $\begin{array}{l}-0.0132 \\
0.2481 * *\end{array}$ & $\begin{array}{l}0.1566 \\
0.0479\end{array}$ & $\begin{array}{l}0.4241^{* *} \\
0.2039^{*}\end{array}$ & $\begin{array}{l}0.1607 \\
0.0208\end{array}$ & $\begin{array}{c}0.0705 \\
0.2148 *\end{array}$ & $\begin{array}{l}0.0026 \\
0.1099\end{array}$ & $\begin{array}{l}0.1802 * \\
-0.0266\end{array}$ & $\begin{array}{c}0.0562 \\
-0.0860\end{array}$ & $\begin{array}{l}0.1183 \\
0.0638\end{array}$ & $\begin{array}{l}0.2428 * * \\
0.2242^{* *}\end{array}$ \\
\hline Leaf length & $\begin{array}{l}\mathbf{G} \\
\mathbf{P}\end{array}$ & & & & & & 1 & $\begin{array}{l}0.6979 * * \\
0.4766 * *\end{array}$ & $\begin{array}{l}-0.2438^{* * *} \\
-0.1761^{*}\end{array}$ & $\begin{array}{l}0.1455 \\
0.1161\end{array}$ & $\begin{array}{l}0.3253^{* *} \\
0.2539^{* * *}\end{array}$ & $\begin{array}{l}0.0820 \\
0.0991\end{array}$ & $\begin{array}{l}0.3418^{* *} \\
0.2411^{* *}\end{array}$ & $\begin{array}{c}0.2289 * * \\
0.1485\end{array}$ & $\begin{array}{l}-0.0603 \\
-0.0684\end{array}$ & $\begin{array}{l}-0.1884^{*} \\
-0.1351\end{array}$ & $\begin{array}{l}0.1257 \\
0.1030\end{array}$ & $\begin{array}{l}-0.0736 \\
-0.0658\end{array}$ & $\begin{array}{c}0.1763 * \\
0.1353\end{array}$ & $\begin{array}{l}0.2208 * \\
* 0.1483\end{array}$ & $\begin{array}{l}-0.1289 \\
-0.0991\end{array}$ \\
\hline Leaf width & $\begin{array}{l}\mathbf{G} \\
\mathbf{P}\end{array}$ & & & & & & & 1 & $\begin{array}{l}-0.0497 \\
-0.0434\end{array}$ & $\begin{array}{l}0.0786 \\
0.0638\end{array}$ & $\begin{array}{l}0.1275 \\
0.1264\end{array}$ & $\begin{array}{l}0.1340 \\
0.1164\end{array}$ & $\begin{array}{l}0.4145^{* * *} \\
0.2254^{* * *}\end{array}$ & $\begin{array}{l}0.3077 * * \\
0.2669 * *\end{array}$ & $\begin{array}{l}-0.0494 \\
-0.0299\end{array}$ & $\begin{array}{l}-0.0288 \\
-0.0648\end{array}$ & $\begin{array}{l}0.1860^{*} \\
0.1591\end{array}$ & $\begin{array}{l}-0.0525 \\
-0.0174\end{array}$ & $\begin{array}{l}0.0971 \\
0.0782\end{array}$ & $\begin{array}{l}-0.0266 \\
-0.0107\end{array}$ & $\begin{array}{l}0.0682 \\
0.0608\end{array}$ \\
\hline $\begin{array}{l}\text { Clusters/ } \\
\text { plant }\end{array}$ & $\begin{array}{l}\mathbf{G} \\
\mathbf{P}\end{array}$ & & & & & & & & 1 & $\begin{array}{l}0.0768 \\
0.0583\end{array}$ & $\begin{array}{l}0.5946^{* * *} \\
0.5775^{* *}\end{array}$ & $\begin{array}{l}0.1119 \\
0.1089\end{array}$ & $\begin{array}{l}-0.0102 \\
-0.0123\end{array}$ & $\begin{array}{c}0.2261^{* *} \\
0.1926^{*}\end{array}$ & $\begin{array}{l}0.2053^{*} \\
0.1823^{*}\end{array}$ & $\begin{array}{l}0.3379 * * \\
0.3166 * *\end{array}$ & $\begin{array}{l}0.1012 \\
0.0895\end{array}$ & $\begin{array}{l}-0.0995 \\
-0.0880\end{array}$ & $\begin{array}{l}-0.1022 \\
-0.1016\end{array}$ & $\begin{array}{l}-0.0833 \\
-0.0902\end{array}$ & $\begin{array}{l}0.3787 * * \\
0.3556^{* *}\end{array}$ \\
\hline $\begin{array}{l}\text { Pods/ } \\
\text { cluster }\end{array}$ & $\begin{array}{l}\mathbf{G} \\
\mathbf{P}\end{array}$ & & & & & & & & & 1 & $\begin{array}{l}0.3131 \text { *** } \\
0.2883^{* * *}\end{array}$ & $\begin{array}{l}-0.1225 \\
-0.1147\end{array}$ & $\begin{array}{l}-0.1325 \\
-0.0981\end{array}$ & $\begin{array}{l}0.0201 \\
0.0164\end{array}$ & $\begin{array}{r}0.1878^{*} \\
0.1460\end{array}$ & $\begin{array}{l}-0.0248 \\
-0.0312\end{array}$ & $\begin{array}{l}0.2911 \text { ** } \\
0.2608^{* *}\end{array}$ & $\begin{array}{l}-0.0209 \\
-0.0131\end{array}$ & $\begin{array}{l}0.0542 \\
0.0470\end{array}$ & $\begin{array}{c}0.2400^{*} \\
* \\
0.2075^{*}\end{array}$ & $\begin{array}{l}0.0838 \\
0.0903\end{array}$ \\
\hline Pods/plant & $\begin{array}{l}\mathbf{G} \\
\mathbf{P}\end{array}$ & & & & & & & & & & 1 & $\begin{array}{l}0.0711 \\
0.0747\end{array}$ & $\begin{array}{l}0.0730 \\
0.0569\end{array}$ & $\begin{array}{l}0.1958^{*} \\
0.1647^{*}\end{array}$ & $\begin{array}{l}0.0832 \\
0.0825\end{array}$ & $\begin{array}{l}0.2607 * * \\
0.2422^{* * *}\end{array}$ & $\begin{array}{l}0.2205^{* *} \\
0.2106^{*}\end{array}$ & $\begin{array}{c}0.2219^{*} \\
* \\
-0.2093^{*}\end{array}$ & $\begin{array}{l}0.0171 \\
0.0181\end{array}$ & $\begin{array}{l}0.0929 \\
0.0181\end{array}$ & $\begin{array}{l}0.3178 * * \\
0.3084 * *\end{array}$ \\
\hline Seeds/pod & $\begin{array}{l}\mathbf{G} \\
\mathbf{P}\end{array}$ & & & & & & & & & & & 1 & $\begin{array}{l}0.3065 \text { ** } \\
0.2518^{* * *}\end{array}$ & $\begin{array}{l}0.0133 \\
0.0171\end{array}$ & $\begin{array}{l}0.0901 \\
0.0849\end{array}$ & $\begin{array}{l}0.0407 \\
0.0397\end{array}$ & $\begin{array}{l}-0.0198 \\
-0.0030\end{array}$ & $\begin{array}{l}0.1778^{*} \\
0.1543\end{array}$ & $\begin{array}{l}-0.1860^{*} \\
-0.1765^{*}\end{array}$ & $\begin{array}{l}-0.0908 \\
-0.0898\end{array}$ & $\begin{array}{l}0.1008 \\
0.1040\end{array}$ \\
\hline Pod length & $\begin{array}{l}\mathbf{G} \\
\mathbf{P}\end{array}$ & & & & & & & & & & & & 1 & $\begin{array}{l}0.5032^{* *} \\
0.3916^{* * *}\end{array}$ & $\begin{array}{c}0.2395^{*} \\
* \\
0.1820^{*}\end{array}$ & $\begin{array}{l}0.1981^{*} \\
0.1681^{*}\end{array}$ & $\begin{array}{l}0.1398 \\
0.1008\end{array}$ & $\begin{array}{l}-0.0613 \\
-0.0369\end{array}$ & $\begin{array}{l}0.1036 \\
0.0824\end{array}$ & $\begin{array}{l}-0.0559 \\
-0.0252\end{array}$ & $\begin{array}{l}0.3049^{* *} \\
0.2537^{* *}\end{array}$ \\
\hline $\begin{array}{l}\text { 100-Seed } \\
\text { weight }\end{array}$ & $\begin{array}{l}\mathbf{G} \\
\mathbf{P}\end{array}$ & & & & & & & & & & & & & 1 & $\begin{array}{c}0.2603^{*} \\
* \\
0.2526^{*} \\
*\end{array}$ & $\begin{array}{l}0.3806^{* * *} \\
0.3077^{* *}\end{array}$ & $\begin{array}{c}0.2078^{* *} \\
0.1847^{*}\end{array}$ & $\begin{array}{l}-0.1031 \\
-0.0431\end{array}$ & $\begin{array}{l}-0.1112 \\
-0.0931\end{array}$ & $\begin{array}{l}0.0531 \\
0.0579\end{array}$ & $\begin{array}{l}0.4248 * * \\
0.3890^{* * *}\end{array}$ \\
\hline Shelling \% & $\begin{array}{l}\mathbf{G} \\
\mathbf{P}\end{array}$ & & & & & & & & & & & & & & 1 & $\begin{array}{l}0.4130^{* * *} \\
0.3722^{* * *}\end{array}$ & $\begin{array}{l}0.2753 * * \\
0.2583^{* *}\end{array}$ & $\begin{array}{l}-0.1562 \\
-0.1109\end{array}$ & $\begin{array}{l}0.3885 * * \\
- \\
0.3524^{* * *}\end{array}$ & $\begin{array}{c}-0.0110 \\
0.0103\end{array}$ & $\begin{array}{l}0.5165 * * \\
0.4929^{* * *}\end{array}$ \\
\hline $\begin{array}{l}\text { Biological } \\
\text { yield/plant }\end{array}$ & $\begin{array}{l}\mathbf{G} \\
\mathbf{P}\end{array}$ & & & & & & & & & & & & & & & 1 & $\begin{array}{l}-0.0268 \\
-0.0440\end{array}$ & $\begin{array}{l}-0.1027 \\
-0.0926\end{array}$ & $\begin{array}{l}-0.2104 \\
-0.1982^{*}\end{array}$ & $\begin{array}{l}-0.1085 \\
-0.1005\end{array}$ & $\begin{array}{l}0.8113^{* * *} \\
0.7497^{* * *}\end{array}$ \\
\hline $\begin{array}{l}\text { Harvest } \\
\text { index }\end{array}$ & $\begin{array}{l}\mathbf{G} \\
\mathbf{P}\end{array}$ & & & & & & & & & & & & & & & & 1 & $\begin{array}{c}-0.1905^{*} \\
-.1681^{*} \\
\end{array}$ & $\begin{array}{l}0.0977 \\
0.0938\end{array}$ & $\begin{array}{l}0.2020^{*} \\
0.2026^{*}\end{array}$ & $\begin{array}{l}0.5009 * * \\
0.4809 * *\end{array}$ \\
\hline $\begin{array}{l}\text { Protein } \\
\text { content }\end{array}$ & $\begin{array}{l}\mathbf{G} \\
\mathbf{P}\end{array}$ & & & & & & & & & & & & & & & & & 1 & $\begin{array}{l}0.0856 \\
0.0766\end{array}$ & $\begin{array}{l}-0.0892 \\
-0.0893\end{array}$ & $\begin{array}{l}-0.2237 \text { ** } \\
-0.1909^{*}\end{array}$ \\
\hline $\begin{array}{l}\text { Calcium } \\
\text { content }\end{array}$ & $\begin{array}{l}\mathbf{G} \\
\mathbf{P}\end{array}$ & & & & & & & & & & & & & & & & & & 1 & $\begin{array}{l}-0.0919 \\
-0.0906\end{array}$ & $\begin{array}{l}-0.1378 \\
-0.1344\end{array}$ \\
\hline $\begin{array}{l}\text { Seed } \\
\text { hardness }\end{array}$ & $\begin{array}{l}\text { G } \\
\mathbf{P}\end{array}$ & & & & & & & & & & & & & & & & & & & 1 & $\begin{array}{l}-0.0086 \\
-0.0010\end{array}$ \\
\hline $\begin{array}{l}\text { Seed } \\
\text { yield/plant }\end{array}$ & $\begin{array}{l}\mathbf{G} \\
\mathbf{P}\end{array}$ & & & & & & & & & & & & & & & & & & & & 1 \\
\hline
\end{tabular}


Table.2 Direct and indirect effects of different traits on seed yield per plant in mung bean genotypes

\begin{tabular}{|c|c|c|c|c|c|c|c|c|c|c|c|c|c|c|c|c|c|c|c|c|}
\hline Character & & $\begin{array}{c}\text { Days to } \\
50 \% \\
\text { flowering }\end{array}$ & $\begin{array}{c}\text { Days to } \\
\text { maturity }\end{array}$ & $\begin{array}{c}\text { Days to } \\
\text { shattering }\end{array}$ & $\begin{array}{c}\text { Plant } \\
\text { height }\end{array}$ & $\begin{array}{c}\text { Primary } \\
\text { branches/plant }\end{array}$ & $\begin{array}{c}\text { Leaf } \\
\text { length }\end{array}$ & $\begin{array}{c}\text { Leaf } \\
\text { width }\end{array}$ & Clusters/plant & Pods/cluster & Pods/plant & Seeds/pod & $\begin{array}{c}\text { Pod } \\
\text { length }\end{array}$ & $\begin{array}{c}\text { 100- } \\
\text { Seed } \\
\text { weight }\end{array}$ & $\begin{array}{c}\text { Shelling } \\
\%\end{array}$ & $\begin{array}{c}\text { Biological } \\
\text { yield/plant }\end{array}$ & $\begin{array}{c}\text { Harvest } \\
\text { index }\end{array}$ & $\begin{array}{l}\text { Protein } \\
\text { content }\end{array}$ & $\begin{array}{l}\text { Calcium } \\
\text { content }\end{array}$ & $\begin{array}{c}\text { Seed } \\
\text { hardness }\end{array}$ \\
\hline $\begin{array}{l}\text { Days to } 50 \% \\
\text { flowering }\end{array}$ & $\begin{array}{l}\text { G } \\
P\end{array}$ & $\begin{array}{l}-0.2726 \\
-0.0816\end{array}$ & $\begin{array}{c}-0.2633 \\
0.0775\end{array}$ & $\begin{array}{l}-0.2647 \\
0.0748\end{array}$ & $\begin{array}{l}-0.3966 \\
0.0240\end{array}$ & $\begin{array}{l}-0.1721 \\
0.0086\end{array}$ & $\begin{array}{l}-0.1005 \\
0.0026\end{array}$ & $\begin{array}{r}-0.4060 \\
0.0228 \\
\end{array}$ & $\begin{array}{l}-0.0952 \\
0.0059\end{array}$ & $\begin{array}{c}0.0359 \\
-0.0026\end{array}$ & $\begin{array}{l}-0.2133 \\
0.0121\end{array}$ & $\begin{array}{c}0.1106 \\
-0.0079\end{array}$ & $\begin{array}{l}-0.3148 \\
0.0155\end{array}$ & $\begin{array}{r}-0.4349 \\
0.0227\end{array}$ & $\begin{array}{c}-0.0080 \\
0.0000\end{array}$ & $\begin{array}{l}-0.2970 \\
0.0169\end{array}$ & $\begin{array}{c}-0.1872 \\
0.0098\end{array}$ & $\begin{array}{c}0.2388 \\
-0.0130\end{array}$ & $\begin{array}{c}0.0137 \\
-0.0007\end{array}$ & $\begin{array}{l}0.1313 \\
-0.0084\end{array}$ \\
\hline $\begin{array}{l}\text { Days to } \\
\text { maturity }\end{array}$ & $\begin{array}{l}\mathrm{G} \\
\mathrm{P} \\
\end{array}$ & $\begin{array}{l}0.3327 \\
0.1134\end{array}$ & $\begin{array}{l}0.3425 \\
0.1193 \\
\end{array}$ & $\begin{array}{l}0.3428 \\
0.1129 \\
\end{array}$ & $\begin{array}{l}0.4746 \\
0.0388 \\
\end{array}$ & $\begin{array}{l}0.1415 \\
0.0102 \\
\end{array}$ & $\begin{array}{l}0.1194 \\
0.0055 \\
\end{array}$ & $\begin{array}{l}0.4265 \\
0.0321 \\
\end{array}$ & $\begin{array}{l}0.0749 \\
0.0059\end{array}$ & $\begin{array}{l}0.0063 \\
0.0003\end{array}$ & $\begin{array}{l}0.2255 \\
0.0195\end{array}$ & $\begin{array}{l}-0.0832 \\
-0.0065 \\
\end{array}$ & $\begin{array}{l}0.3282 \\
0.0274 \\
\end{array}$ & $\begin{array}{l}0.4000 \\
0.0300 \\
\end{array}$ & $\begin{array}{l}0.0214 \\
0.0023 \\
\end{array}$ & $\begin{array}{l}0.2789 \\
0.0222\end{array}$ & $\begin{array}{l}0.2195 \\
0.0150 \\
\end{array}$ & $\begin{array}{l}-0.1615 \\
-0.0123 \\
\end{array}$ & $\begin{array}{l}0.0256 \\
0.0033 \\
\end{array}$ & $\begin{array}{l}-0.1506 \\
-0.0145\end{array}$ \\
\hline $\begin{array}{l}\text { Days to } \\
\text { shattering }\end{array}$ & $\begin{array}{l}\text { G } \\
P\end{array}$ & $\begin{array}{r}0.0139 \\
-0.0825\end{array}$ & $\begin{array}{r}0.0140 \\
-0.0851\end{array}$ & $\begin{array}{r}0.0140 \\
-0.0899\end{array}$ & $\begin{array}{c}0.0041 \\
-0.0237\end{array}$ & $\begin{array}{c}0.0028 \\
-0.0146\end{array}$ & $\begin{array}{r}0.0009 \\
-0.0047\end{array}$ & $\begin{array}{c}0.0052 \\
-0.0226\end{array}$ & $\begin{array}{c}0.0018 \\
-0.0107\end{array}$ & $\begin{array}{c}0.0002 \\
-0.0019\end{array}$ & $\begin{array}{c}0.0029 \\
-0.0164\end{array}$ & $\begin{array}{l}-0.0006 \\
0.0028\end{array}$ & $\begin{array}{c}0.0017 \\
-0.0154\end{array}$ & $\begin{array}{c}0.0043 \\
-0.0205\end{array}$ & $\begin{array}{l}0.0002 \\
0.0001\end{array}$ & $\begin{array}{c}0.0032 \\
-0.0199\end{array}$ & $\begin{array}{c}0.0021 \\
-0.0104\end{array}$ & $\begin{array}{l}-0.0016 \\
0.0086\end{array}$ & $\begin{array}{c}-0.0004 \\
0.0022\end{array}$ & $\begin{array}{l}-0.0016 \\
0.0107\end{array}$ \\
\hline Plant height & $\begin{array}{l}\text { G } \\
P\end{array}$ & $\begin{array}{l}-0.0146 \\
-0.0038\end{array}$ & $\begin{array}{l}-0.0166 \\
-0.0043\end{array}$ & $\begin{array}{l}-0.0140 \\
-0.0034\end{array}$ & $\begin{array}{r}-0.0470 \\
-0.0131\end{array}$ & $\begin{array}{l}-0.0006 \\
-0.0001\end{array}$ & $\begin{array}{l}-0.0093 \\
-0.0022\end{array}$ & $\begin{array}{l}-0.0264 \\
-0.0063\end{array}$ & $\begin{array}{l}0.0066 \\
0.0018\end{array}$ & $\begin{array}{l}-0.0003 \\
-0.0001\end{array}$ & $\begin{array}{l}0.0006 \\
0.0002\end{array}$ & $\begin{array}{l}-0.0074 \\
-0.0020\end{array}$ & $\begin{array}{l}-0.0199 \\
-0.0042\end{array}$ & $\begin{array}{l}-0.0076 \\
-0.0018\end{array}$ & $\begin{array}{l}-0.0033 \\
-0.0008\end{array}$ & $\begin{array}{l}-0.0001 \\
0.0000\end{array}$ & $\begin{array}{l}-0.0085 \\
-0.0022\end{array}$ & $\begin{array}{l}-0.0026 \\
-0.0006\end{array}$ & $\begin{array}{l}-0.0056 \\
-0.0015\end{array}$ & $\begin{array}{l}0.0063 \\
0.0017\end{array}$ \\
\hline $\begin{array}{l}\text { Primary } \\
\text { branches/plant }\end{array}$ & $\begin{array}{l}\text { G } \\
P\end{array}$ & $\begin{array}{c}0.0005 \\
-0.0014\end{array}$ & $\begin{array}{c}0.0004 \\
-0.0012\end{array}$ & $\begin{array}{l}0.0007 \\
-0.0022\end{array}$ & $\begin{array}{c}0.0001 \\
-0.0001\end{array}$ & $\begin{array}{c}0.0038 \\
-0.0135\end{array}$ & $\begin{array}{r}0.0007 \\
-0.0024\end{array}$ & $\begin{array}{r}0.0080 \\
-0.0022\end{array}$ & $\begin{array}{c}0.0012 \\
-0.0040\end{array}$ & $\begin{array}{l}0.0003 \\
0.0010\end{array}$ & $\begin{array}{c}0.0012 \\
-0.0038\end{array}$ & $\begin{array}{c}0.0010 \\
-0.0034\end{array}$ & $\begin{array}{c}0.0004 \\
-0.0006\end{array}$ & $\begin{array}{c}0.0009 \\
-0.0028\end{array}$ & $\begin{array}{c}0.0001 \\
-0.0003\end{array}$ & $\begin{array}{c}0.0009 \\
-0.0029\end{array}$ & $\begin{array}{c}0.0005 \\
-0.0015\end{array}$ & $\begin{array}{l}-0.0001 \\
0.0004\end{array}$ & $\begin{array}{c}-0.0003 \\
0.0012\end{array}$ & $\begin{array}{c}0.0003 \\
-0.0098\end{array}$ \\
\hline Leaf length & $\begin{array}{l}\mathrm{G} \\
\mathrm{P}\end{array}$ & $\begin{array}{l}-0.0026 \\
-0.0021\end{array}$ & $\begin{array}{l}-0.0029 \\
-0.0031\end{array}$ & $\begin{array}{l}-0.0021 \\
-0.0034\end{array}$ & $\begin{array}{l}-0.0065 \\
-0.0112\end{array}$ & $\begin{array}{l}-0.0061 \\
-0.0116\end{array}$ & $\begin{array}{l}-0.0328 \\
-0.0664\end{array}$ & $\begin{array}{l}-0.0229 \\
-0.0317\end{array}$ & $\begin{array}{l}0.0080 \\
0.0117\end{array}$ & $\begin{array}{l}-0.0048 \\
-0.0077\end{array}$ & $\begin{array}{l}-0.0107 \\
-0.0169\end{array}$ & $\begin{array}{l}-0.0027 \\
-0.0066\end{array}$ & $\begin{array}{l}-0.0112 \\
-0.0160\end{array}$ & $\begin{array}{l}-0.0075 \\
-0.0099\end{array}$ & $\begin{array}{l}0.0020 \\
0.0045\end{array}$ & $\begin{array}{l}0.0062 \\
0.0090\end{array}$ & $\begin{array}{r}-0.0041 \\
-0.0068\end{array}$ & $\begin{array}{l}0.0024 \\
0.0044\end{array}$ & $\begin{array}{l}-0.0058 \\
-0.0090\end{array}$ & $\begin{array}{l}-0.0072 \\
-0.0002\end{array}$ \\
\hline Leaf width & $\begin{array}{l}\text { G } \\
P\end{array}$ & $\begin{array}{l}-0.0016 \\
0.0060\end{array}$ & $\begin{array}{c}-0.0016 \\
0.0057\end{array}$ & $\begin{array}{c}-0.0018 \\
0.0054\end{array}$ & $\begin{array}{l}-0.0028 \\
0.0102\end{array}$ & $\begin{array}{l}-0.0011 \\
0.0034\end{array}$ & $\begin{array}{l}-0.0034 \\
0.0102\end{array}$ & $\begin{array}{c}-0.0049 \\
0.0214\end{array}$ & $\begin{array}{c}0.0002 \\
-0.0009\end{array}$ & $\begin{array}{c}-0.0004 \\
0.0014\end{array}$ & $\begin{array}{c}-0.0006 \\
0.0027\end{array}$ & $\begin{array}{l}-0.0007 \\
0.0025\end{array}$ & $\begin{array}{c}-0.0020 \\
0.0048\end{array}$ & $\begin{array}{r}-0.0015 \\
0.0057\end{array}$ & $\begin{array}{c}0.0002 \\
-0.0006\end{array}$ & $\begin{array}{c}0.0001 \\
-0.0014\end{array}$ & $\begin{array}{c}-0.0009 \\
0.0034\end{array}$ & $\begin{array}{r}0.0003 \\
-0.0004\end{array}$ & $\begin{array}{r}-0.0005 \\
0.0017\end{array}$ & $\begin{array}{c}0.0001 \\
-0.0064\end{array}$ \\
\hline Clusters/plant & $\begin{array}{l}\text { G } \\
P\end{array}$ & $\begin{array}{l}0.0080 \\
0.0051\end{array}$ & $\begin{array}{l}0.0059 \\
0.0035\end{array}$ & $\begin{array}{l}0.0133 \\
0.0084\end{array}$ & $\begin{array}{l}-0.0148 \\
-0.0097\end{array}$ & $\begin{array}{l}0.0325 \\
0.0207\end{array}$ & $\begin{array}{r}-0.0259 \\
-0.0125\end{array}$ & $\begin{array}{l}-0.0053 \\
-0.0031\end{array}$ & $\begin{array}{l}0.1064 \\
0.0708\end{array}$ & $\begin{array}{l}0.0082 \\
0.0041\end{array}$ & $\begin{array}{l}0.0632 \\
0.0409\end{array}$ & $\begin{array}{l}0.0119 \\
0.0077\end{array}$ & $\begin{array}{l}-0.0011 \\
-0.0009\end{array}$ & $\begin{array}{l}0.0240 \\
0.0136\end{array}$ & $\begin{array}{l}0.0218 \\
0.0129\end{array}$ & $\begin{array}{l}0.0359 \\
0.0224\end{array}$ & $\begin{array}{l}0.0108 \\
0.0063\end{array}$ & $\begin{array}{l}-0.0106 \\
-0.0062\end{array}$ & $\begin{array}{l}-0.0109 \\
-0.0072\end{array}$ & $\begin{array}{c}-0.0089 \\
0.0005\end{array}$ \\
\hline Pods/cluster & $\begin{array}{l}\text { G } \\
P\end{array}$ & $\begin{array}{c}0.0012 \\
-0.0001\end{array}$ & $\begin{array}{c}-0.0002 \\
0.0000\end{array}$ & $\begin{array}{c}-0.0006 \\
0.0001\end{array}$ & $\begin{array}{l}-0.0002 \\
0.0000\end{array}$ & $\begin{array}{l}-0.0030 \\
0.0002\end{array}$ & $\begin{array}{l}-0.006 \\
0.0003\end{array}$ & $\begin{array}{c}-0.0033 \\
0.0001\end{array}$ & $\begin{array}{c}-0.0032 \\
0.0001\end{array}$ & $\begin{array}{l}0.0414 \\
0.0023\end{array}$ & $\begin{array}{c}-0.0130 \\
0.0007\end{array}$ & $\begin{array}{c}0.0051 \\
-0.0003\end{array}$ & $\begin{array}{c}0.0055 \\
-0.0002\end{array}$ & $\begin{array}{c}-0.0008 \\
0.0000\end{array}$ & $\begin{array}{c}-0.0078 \\
0.0003\end{array}$ & $\begin{array}{c}0.0010 \\
-0.0001\end{array}$ & $\begin{array}{c}-0.0121 \\
0.0006\end{array}$ & $\begin{array}{l}0.0009 \\
0.0000\end{array}$ & $\begin{array}{c}-0.0022 \\
0.0001\end{array}$ & $\begin{array}{l}-0.0099 \\
-0.0018\end{array}$ \\
\hline Pods/plant & $\begin{array}{l}\mathrm{G} \\
\mathrm{P}\end{array}$ & $\begin{array}{l}-0.0157 \\
-0.0031\end{array}$ & $\begin{array}{l}-0.0157 \\
-0.0034\end{array}$ & $\begin{array}{l}-0.0194 \\
-0.0038\end{array}$ & $\begin{array}{l}0.0012 \\
0.0003\end{array}$ & $\begin{array}{l}-0.0292 \\
-0.0059\end{array}$ & $\begin{array}{l}-0.0304 \\
-0.0053\end{array}$ & $\begin{array}{l}-0.0119 \\
-0.0027\end{array}$ & $\begin{array}{l}-0.0556 \\
-0.0122\end{array}$ & $\begin{array}{l}-0.0293 \\
-0.0061\end{array}$ & $\begin{array}{l}0.0934 \\
0.0211\end{array}$ & $\begin{array}{l}-0.0066 \\
-0.0016\end{array}$ & $\begin{array}{l}-0.0068 \\
-0.0012\end{array}$ & $\begin{array}{l}-0.0183 \\
-0.0035\end{array}$ & $\begin{array}{l}-0.0078 \\
-0.0017\end{array}$ & $\begin{array}{l}-0.0244 \\
-0.0051\end{array}$ & $\begin{array}{l}-0.0206 \\
-0.0044\end{array}$ & $\begin{array}{l}0.0207 \\
0.0044\end{array}$ & $\begin{array}{l}-0.0016 \\
-0.0004\end{array}$ & $\begin{array}{l}-0.0087 \\
-0.0078\end{array}$ \\
\hline Seeds/pod & $\begin{array}{l}\mathrm{G} \\
\mathrm{P}\end{array}$ & $\begin{array}{l}-0.0044 \\
-0.0084\end{array}$ & $\begin{array}{l}-0.0031 \\
-0.0047\end{array}$ & $\begin{array}{l}-0.0021 \\
-0.0027\end{array}$ & $\begin{array}{l}0.0079 \\
0.0135\end{array}$ & $\begin{array}{l}0.0129 \\
0.0214\end{array}$ & $\begin{array}{l}0.0042 \\
0.0086\end{array}$ & $\begin{array}{l}0.0068 \\
0.0101\end{array}$ & $\begin{array}{l}0.0057 \\
0.0094\end{array}$ & $\begin{array}{l}-0.0062 \\
-0.0099\end{array}$ & $\begin{array}{l}0.0036 \\
0.0065\end{array}$ & $\begin{array}{l}0.0507 \\
0.0864\end{array}$ & $\begin{array}{l}0.0155 \\
0.0218\end{array}$ & $\begin{array}{l}0.0007 \\
0.0015\end{array}$ & $\begin{array}{l}0.0046 \\
0.0073\end{array}$ & $\begin{array}{l}0.0021 \\
0.0034\end{array}$ & $\begin{array}{l}-0.0010 \\
-0.0003\end{array}$ & $\begin{array}{l}0.0090 \\
0.0133\end{array}$ & $\begin{array}{l}-0.0094 \\
-0.0153\end{array}$ & $\begin{array}{l}-0.0046 \\
-0.0009\end{array}$ \\
\hline Pod length & $\begin{array}{l}\text { G } \\
P\end{array}$ & $\begin{array}{l}0.0195 \\
0.0066\end{array}$ & $\begin{array}{l}0.0193 \\
0.0080\end{array}$ & $\begin{array}{l}0.0097 \\
0.0059\end{array}$ & $\begin{array}{l}0.0334 \\
0.0112\end{array}$ & $\begin{array}{l}0.0081 \\
0.0017\end{array}$ & $\begin{array}{l}0.0269 \\
0.0084\end{array}$ & $\begin{array}{l}0.0327 \\
0.0078\end{array}$ & $\begin{array}{l}-0.0008 \\
-0.0004\end{array}$ & $\begin{array}{l}-0.0104 \\
-0.0034\end{array}$ & $\begin{array}{l}0.0058 \\
0.0020\end{array}$ & $\begin{array}{l}0.0241 \\
0.0087\end{array}$ & $\begin{array}{l}0.0788 \\
0.0347\end{array}$ & $\begin{array}{l}0.0396 \\
0.0136\end{array}$ & $\begin{array}{l}0.0189 \\
0.0063\end{array}$ & $\begin{array}{l}0.0156 \\
0.0058\end{array}$ & $\begin{array}{l}0.0110 \\
0.0035\end{array}$ & $\begin{array}{l}-0.0048 \\
-0.0013\end{array}$ & $\begin{array}{l}0.0082 \\
0.0029\end{array}$ & $\begin{array}{c}-0.0044 \\
0.0013\end{array}$ \\
\hline $\begin{array}{l}\text { 100-Seed } \\
\text { weight }\end{array}$ & $\begin{array}{l}\mathrm{G} \\
\mathrm{P}\end{array}$ & $\begin{array}{l}0.0039 \\
0.0064\end{array}$ & $\begin{array}{l}0.0034 \\
0.0058\end{array}$ & $\begin{array}{l}0.0035 \\
0.0053\end{array}$ & $\begin{array}{l}0.0018 \\
0.0032\end{array}$ & $\begin{array}{l}0.0028 \\
0.0047\end{array}$ & $\begin{array}{l}0.0026 \\
0.0034\end{array}$ & $\begin{array}{l}0.0035 \\
0.0062\end{array}$ & $\begin{array}{l}0.0026 \\
0.0045\end{array}$ & $\begin{array}{l}0.0002 \\
0.0004\end{array}$ & $\begin{array}{l}0.0023 \\
0.0038\end{array}$ & $\begin{array}{l}0.0002 \\
0.0004\end{array}$ & $\begin{array}{l}0.0058 \\
0.0091\end{array}$ & $\begin{array}{l}0.0115 \\
0.0231\end{array}$ & $\begin{array}{l}0.0030 \\
0.0058\end{array}$ & $\begin{array}{l}0.0044 \\
0.0071\end{array}$ & $\begin{array}{l}0.0024 \\
0.0043\end{array}$ & $\begin{array}{l}-0.0012 \\
-0.001\end{array}$ & $\begin{array}{l}-0.0013 \\
-0.0022\end{array}$ & $\begin{array}{l}0.0006 \\
0.0009\end{array}$ \\
\hline Shelling \% & $\begin{array}{l}\mathrm{G} \\
\mathrm{P}\end{array}$ & $\begin{array}{c}-0.0001 \\
0.0000\end{array}$ & $\begin{array}{c}-0.0003 \\
0.0016\end{array}$ & $\begin{array}{l}-0.0002 \\
-0.0001\end{array}$ & $\begin{array}{c}-0.0013 \\
0.0050\end{array}$ & $\begin{array}{c}-0.0003 \\
0.0018\end{array}$ & $\begin{array}{c}0.0011 \\
-0.0058\end{array}$ & $\begin{array}{c}0.0009 \\
-0.0025\end{array}$ & $\begin{array}{c}-0.0038 \\
0.0155\end{array}$ & $\begin{array}{c}-0.0035 \\
0.0124\end{array}$ & $\begin{array}{c}-0.0015 \\
0.0070\end{array}$ & $\begin{array}{c}-0.0017 \\
0.0072\end{array}$ & $\begin{array}{c}-0.0044 \\
0.0155\end{array}$ & $\begin{array}{c}-0.0048 \\
0.0215\end{array}$ & $\begin{array}{c}-0.0185 \\
0.0851\end{array}$ & $\begin{array}{c}-0.0076 \\
0.0317\end{array}$ & $\begin{array}{c}-0.0051 \\
0.0220\end{array}$ & $\begin{array}{c}0.0029 \\
-0.0094\end{array}$ & $\begin{array}{c}0.0072 \\
-0.0300\end{array}$ & $\begin{array}{c}0.0002 \\
-0.0685\end{array}$ \\
\hline $\begin{array}{l}\text { Biological } \\
\text { yield/plant }\end{array}$ & $\begin{array}{l}\mathrm{G} \\
\mathrm{P}\end{array}$ & $\begin{array}{l}0.1841 \\
0.1412\end{array}$ & $\begin{array}{l}0.1638 \\
0.1267\end{array}$ & $\begin{array}{l}0.1784 \\
0.1505\end{array}$ & $\begin{array}{l}0.0020 \\
0.0015\end{array}$ & $\begin{array}{l}0.1801 \\
0.1463\end{array}$ & $\begin{array}{l}-0.1485 \\
-0.0920\end{array}$ & $\begin{array}{l}-0.0227 \\
-0.0441\end{array}$ & $\begin{array}{l}0.2665 \\
0.2157\end{array}$ & $\begin{array}{l}-0.0196 \\
-0.0213\end{array}$ & $\begin{array}{l}0.2055 \\
0.1650\end{array}$ & $\begin{array}{l}0.0321 \\
0.0270\end{array}$ & $\begin{array}{l}0.1562 \\
0.1145\end{array}$ & $\begin{array}{l}0.3001 \\
0.2097\end{array}$ & $\begin{array}{l}0.3256 \\
0.2536\end{array}$ & $\begin{array}{l}0.7885 \\
0.6813\end{array}$ & $\begin{array}{l}-0.0211 \\
-0.0300\end{array}$ & $\begin{array}{l}-0.0810 \\
-0.0631\end{array}$ & $\begin{array}{l}-0.1659 \\
-0.1350\end{array}$ & $\begin{array}{c}-0.0855 \\
0.0937\end{array}$ \\
\hline Harvest index & $\begin{array}{l}\text { G } \\
P\end{array}$ & $\begin{array}{l}0.0716 \\
0.0554\end{array}$ & $\begin{array}{l}0.0796 \\
0.0582\end{array}$ & $\begin{array}{l}0.0715 \\
0.0535\end{array}$ & $\begin{array}{l}0.0877 \\
0.0776\end{array}$ & $\begin{array}{l}0.0625 \\
0.0508\end{array}$ & $\begin{array}{l}0.0612 \\
0.0476\end{array}$ & $\begin{array}{l}0.0905 \\
0.0736\end{array}$ & $\begin{array}{l}0.0492 \\
0.0414\end{array}$ & $\begin{array}{l}0.1417 \\
0.1206\end{array}$ & $\begin{array}{l}0.1073 \\
0.0974\end{array}$ & $\begin{array}{l}-0.0097 \\
-0.0014\end{array}$ & $\begin{array}{l}0.0681 \\
0.0466\end{array}$ & $\begin{array}{l}0.1011 \\
0.0854\end{array}$ & $\begin{array}{l}0.1340 \\
0.1194\end{array}$ & $\begin{array}{l}-0.0130 \\
-0.0203\end{array}$ & $\begin{array}{l}0.4867 \\
0.4623\end{array}$ & $\begin{array}{l}-0.0927 \\
-0.0777\end{array}$ & $\begin{array}{l}0.0476 \\
0.0433\end{array}$ & $\begin{array}{l}0.0983 \\
0.0034\end{array}$ \\
\hline $\begin{array}{l}\text { Protein } \\
\text { content }\end{array}$ & $\begin{array}{l}G \\
P\end{array}$ & $\begin{array}{l}0.0260 \\
0.0060\end{array}$ & $\begin{array}{l}0.0167 \\
0.0039\end{array}$ & $\begin{array}{l}0.0157 \\
0.0036\end{array}$ & $\begin{array}{l}-0.0078 \\
-0.0017\end{array}$ & $\begin{array}{l}0.0043 \\
0.0010\end{array}$ & $\begin{array}{l}0.0102 \\
0.0025\end{array}$ & $\begin{array}{l}0.0073 \\
0.0007\end{array}$ & $\begin{array}{l}0.0138 \\
0.0033\end{array}$ & $\begin{array}{l}0.0029 \\
0.0005\end{array}$ & $\begin{array}{l}0.0308 \\
0.0079\end{array}$ & $\begin{array}{l}-0.0247 \\
-0.0058\end{array}$ & $\begin{array}{l}0.0085 \\
0.0014\end{array}$ & $\begin{array}{l}0.0143 \\
0.0016\end{array}$ & $\begin{array}{l}0.0217 \\
0.0042\end{array}$ & $\begin{array}{l}0.0143 \\
0.0035\end{array}$ & $\begin{array}{l}0.0264 \\
0.0063\end{array}$ & $\begin{array}{l}-0.1387 \\
-0.0375\end{array}$ & $\begin{array}{l}-0.0119 \\
-0.0029\end{array}$ & $\begin{array}{c}0.0124 \\
-0.0014\end{array}$ \\
\hline $\begin{array}{l}\text { Calcium } \\
\text { content }\end{array}$ & $\begin{array}{l}\mathrm{G} \\
\mathrm{P}\end{array}$ & $\begin{array}{c}0.0002 \\
-0.0001\end{array}$ & $\begin{array}{c}-0.0004 \\
0.0004\end{array}$ & $\begin{array}{c}0.0007 \\
-0.0004\end{array}$ & $\begin{array}{c}-0.0026 \\
0.0018\end{array}$ & $\begin{array}{c}0.0019 \\
-0.0013\end{array}$ & $\begin{array}{c}-0.0039 \\
0.0021\end{array}$ & $\begin{array}{c}-0.0022 \\
0.0012\end{array}$ & $\begin{array}{c}0.0023 \\
-0.0016\end{array}$ & $\begin{array}{c}-0.0012 \\
0.0007\end{array}$ & $\begin{array}{c}-0.0004 \\
0.0003\end{array}$ & $\begin{array}{c}0.0041 \\
-0.0028\end{array}$ & $\begin{array}{c}-0.0023 \\
0.0013\end{array}$ & $\begin{array}{c}0.0025 \\
-0.0015\end{array}$ & $\begin{array}{c}0.0087 \\
-0.0055\end{array}$ & $\begin{array}{c}0.0047 \\
-0.0031\end{array}$ & $\begin{array}{c}-0.0022 \\
0.0015\end{array}$ & $\begin{array}{c}-0.0019 \\
0.0012\end{array}$ & $\begin{array}{l}-\mathbf{- 0 . 0 2 2 3} \\
\mathbf{0 . 0 1 5 7}\end{array}$ & $\begin{array}{l}0.0020 \\
0.0076\end{array}$ \\
\hline Seed hardness & $\begin{array}{l}\text { G } \\
P\end{array}$ & $\begin{array}{l}-0.0022 \\
-0.0008\end{array}$ & $\begin{array}{l}-0.0024 \\
-0.0009\end{array}$ & $\begin{array}{r}-0.0025 \\
-0.0009\end{array}$ & $\begin{array}{l}-0.0028 \\
-0.0010\end{array}$ & $\begin{array}{l}0.0018 \\
0.0005\end{array}$ & $\begin{array}{l}0.0047 \\
0.0011\end{array}$ & $\begin{array}{l}-0.0006 \\
-0.0001\end{array}$ & $\begin{array}{l}-0.0018 \\
-0.0007\end{array}$ & $\begin{array}{l}0.0051 \\
0.0016\end{array}$ & $\begin{array}{l}0.0020 \\
0.0007\end{array}$ & $\begin{array}{l}-0.0019 \\
-0.0007\end{array}$ & $\begin{array}{l}-0.0012 \\
-0.0002\end{array}$ & $\begin{array}{l}0.0011 \\
0.0004\end{array}$ & $\begin{array}{c}-0.0002 \\
0.0001\end{array}$ & $\begin{array}{l}-0.0023 \\
-0.0008\end{array}$ & $\begin{array}{l}0.0043 \\
0.0015\end{array}$ & $\begin{array}{l}-0.0019 \\
-0.0007\end{array}$ & $\begin{array}{l}-0.0020 \\
-0.0007\end{array}$ & $\begin{array}{c}0.0212 \\
-0.0010\end{array}$ \\
\hline $\begin{array}{l}\text { Seed } \\
\text { yield/plant }\end{array}$ & $\begin{array}{l}\mathrm{G} \\
\mathrm{P}\end{array}$ & $\begin{array}{l}0.3477 * * \\
0.3192^{* * *}\end{array}$ & $\begin{array}{l}0.3391 * * \\
0.3080^{* * *}\end{array}$ & $\begin{array}{l}0.3431 * * \\
0.3135^{* *}\end{array}$ & $\begin{array}{l}0.1304 \\
0.1268\end{array}$ & $\begin{array}{l}0.2428^{* *} * \\
0.2242^{* *}\end{array}$ & $\begin{array}{l}-0.1289 \\
-0.0991\end{array}$ & $\begin{array}{l}0.0682 \\
0.0608 \\
\end{array}$ & $\begin{array}{l}0.3787 * * \\
0.3556^{* * *}\end{array}$ & $\begin{array}{l}0.0838 \\
0.0903\end{array}$ & $\begin{array}{l}0.3178 * * \\
0.3084 * *\end{array}$ & $\begin{array}{l}0.1008 \\
0.1040\end{array}$ & $\begin{array}{l}0.3049 * * \\
0.2537 * *\end{array}$ & $\begin{array}{l}0.4248 * * \\
0.3890^{* * *}\end{array}$ & $\begin{array}{l}0.5165 * * \\
0.4929^{* * *}\end{array}$ & $\begin{array}{l}0.8111^{* *} \\
0.7497 * *\end{array}$ & $\begin{array}{l}0.5009^{* *} \\
0.4809^{* *}\end{array}$ & $\begin{array}{c}-0.2237 * * * \\
-0.1909 *\end{array}$ & $\begin{array}{l}-0.1378 \\
-0.1344\end{array}$ & $\begin{array}{l}-0.0086 \\
-0.0010\end{array}$ \\
\hline
\end{tabular}

Diagonal values indicate direct effects

Residual effect at genotypic level $=0.1860$

Residual effect at phenotypic level $=0.3693$ 


\section{Plant height}

At genotypic level, this trait showed nonsignificant positive association (0.1304) and negative direct effect $(-0.0470)$ on seed yield per plant. It also exhibited positive indirect effects via., days to maturity (0.4746), pod length (0.334), harvest index (0.0877), days to shattering (0.0041), primary branches per plant (0.0001), pods per plant (0.0012), seeds per pod (0.0079), 100-seed weight (0.0018), biological yield per plant (0.002) and negative indirect effects via., days to $50 \%$ flowering ($0.3966)$, leaf length $(-0.0065)$, leaf width ($0.0028)$, clusters per plant $(-0.0148)$, pods per cluster (-0.0148), pods per cluster (-0.0002), shelling \% (-0.0015), protein content ($0.0078)$, calcium content $(-0.0026)$ and seed hardness (-0.0028) at genotypic level.

This trait showed non-significant positive association (0.1268) and negative direct effect $(-0.0131)$ on seed yield per plant at phenotypic level. It also showed positive indirect effects via., days to $50 \%$ flowering $(0.0240)$, days to maturity (0.0388), leaf width $(0.0102)$, pods per plant $(0.0003)$, seeds per pod (0.0135), pod length (0.0112), 100seed weight (0.0032), shelling \% (0.005), biological yield per plant (0.0015), harvest index (0.0776) and calcium content (0.0018) and negative indirect effects via., days to shattering (-0.0237), primary branches per plant $(-0.0001)$, leaf length $(0-0.172)$, clusters per plant $(-0.0097)$, protein content $(-0.0017)$ and seed hardness (-0.001). Haritha and Reddy (2002), Saifullah et al., (2002), Mallikarjuna et al., (2006), Reddy et al., (2011) and Kumar and Katiyar (2015) showed similar results for plant height.

\section{Primary branches per plant}

At genotypic level, this trait showed significant positive association $\left(0.2428^{* * *}\right)$ and positive direct effect $(0.0038)$ on seed yield per plant. It also exhibited positive indirect effects via., days to maturity (0.1415), days to shattering (0.0028), clusters per plant (0.0325), seeds per pod (0.0129), pod length (0.0081), 100-seed weight (0.0028), biological yield per plant $(0.1801)$, harvest index (0.0625), protein content (0.0043), calcium content $(0.0019)$ and seed hardness (0.0018) and negative indirect effects via., days to $50 \%$ flowering (-0.1721), plant height $(-0.0006)$, leaf length $(-0.0061)$, leaf width ($0.0011)$, pods per cluster $(-0.003)$, pods per plant $(-0.0292)$ and shelling \% (-0.0003). The trait showed significant positive association $\left(0.2242^{* *}\right)$ and negative direct effect $(-0.0135)$ on seed yield per plant at phenotypic level. It also showed positive indirect effect via., days to $50 \%$ flowering (0.0086), days to maturity (0.0102), leaf width (0.0034), clusters per plant (0.0207), pods per cluster (0.0002), seeds per pod (0.0214), pod length (0.0017), 100-seed weight (0.0047), shelling \% (0.0018), biological yield per plant (0.1463), harvest index (0.0508), protein content (0.0010) and seed hardness (0.0005), while negative indirect effects via., days to shattering $(-0.0146)$, plant height $(-0.0001)$, leaf length $(-0.0116)$, pods per plant $(-0.0059)$ and calcium content $(-0.0013)$. Thus this trait showed significant positive association with positive direct effects at genotypic level and significant positive association with negative direct effects at phenotypic level.

\section{Leaf length}

At genotypic level, this trait showed nonsignificant negative association (-0.1289) and negative direct effect on seed yield per plant. It has showed positive indirect effect via., days to maturity (0.1194), pod length (0.0269), harvest index (0.0612), protein content (0.0102), seed hardness (0.0047), seeds per pod (0.0042), days to shattering (0.0009), primary branches per plant (0.0007), 100 -seed weight $(0.0026)$ and shelling \% 
(0.0011). The trait showed non-significant negative association (-0.0991) and negative direct effects $(-0.0664)$ on seed yield per plant at phenotypic level.

It also showed positive indirect effects via., days to $50 \%$ flowering (0.0026), days to maturity (0.0055), leaf width (0.0102), pods per cluster (0.0003), seeds per pod (0.0086), pod length (0.0084), 100-seed weight $(0.0034)$, harvest index $(0.0476)$ and protein content (0.0011) while negative indirect effects via., days to shattering (-0.0047), plant height $(-0.0022)$, primary branches per plant $(-0.0024)$, clusters per plant $(-0.0125)$, pods per plant $(-0.0053)$, shelling \% (-0.0058) and biological yield per plant (-0.092). Negative indirect effects at genotypic level is showed via., days to $50 \%$ flowering (-0.4060), plant height (-0.0264), leaf length (-0.0229), clusters per plant $(-0.0053)$, pods per cluster $(-$ $0.0033)$, pods per plant (-0.0119), biological yield per plant $(-0.0227)$, calcium content ($0.0022)$ and seed hardness (-0.0006).

\section{Leaf width}

At genotypic level, this trait showed nonsignificant positive association (0.0682) and negative direct effect $(-0.0049)$ on seed yield per plant. It also exhibited positive indirect effects via., days to maturity (0.4265), days to shattering (0.0052), primary branches per plant (0.0080), seeds per pod (0.0068), pod length (0.0327), 100-seed weight (0.0035), shelling \% (0.0009), harvest index (0.0905), protein content $(0.0073)$, while negative indirect effects via., days to $50 \%$ flowering ($0.4060)$, plant height $(-0.0264)$, leaf length ($0.0229)$, clusters per plant $(-0.0053)$, pods per cluster (-0.0033), pods per plant (-0.0119), biological yield per plant (-0.0227), calcium content (-0.0022) and seed hardness (0.0006). This trait also showed nonsignificant positive association (0.0608) and positive direct effects (0.0214) on seed yield per plant at phenotypic level. It also showed positive indirect effects via., days to maturity (0.0321), plant height $(-0.0093)$, pods per cluster $(0.0001)$, seeds per pod $(0.0101)$, pod length (0.0078), 100- seed weight (0.0062), harvest index (0.0736), protein content (0.0007) and calcium content (0.0012), while negative indirect effects via., days to shattering (-0.0226), plant height (-0.063), primary branches per plant $(-0.0022)$, leaf length (-0.0317), clusters per plant (-0.0031), pods per plant (-0.0027), shelling \% ($0.0025)$, biological yield per plant $(-0.0441)$ and seed hardness $(-0.0001)$.

\section{Pods per cluster}

At genotypic level, this trait showed nonsignificant positive association (0.0838) and positive direct effect $(0.0414)$ on seed yield per plant. It also exhibited positive indirect effects via., harvest index (0.1417), days to $50 \%$ flowering (0.0359), days to maturity $(0.0063)$, days to shattering $(0.0002)$, primary branches per plant (0.0003), clusters per plant (0.0082), 100-seed weight $(0.0002)$, protein content (0.0029) and seed hardness (0.0051) and negative indirect effects via., plant height $(-0.003)$, leaf length $(-0.0048)$, leaf width ($0.0004)$, pods per plant $(-0.0293)$, seeds per pod (-0.0062), pod length (-0.0104), shelling $\%$ (-0.0035), biological yield per plant ($0.0196)$ and calcium content (-0.0012).

This trait also showed non-significant positive association (0.0903) and positive direct effects (0.0023) on seed yield per plant at phenotypic level. It also showed positive indirect effects via., harvest index (0.1206), protein content $(0.0005)$, calcium content $(0.0007)$, seed hardness $(0.0016)$, shelling \% (0.0124), 100-seed weight (0.0004), clusters per plant (0.0041), leaf width (0.0014), primary branches per plant (0.0010) and days to maturity (0.0003), while negative indirect effects via., days to $50 \%$ flowering (-0.0026), 
days to shattering (-0.0019), plant height ($0.0001)$, leaf length $(-0.0077)$, pods per plant $(-0.0061)$, seeds per pod (-0.0099), pod length $(-0.0034)$, biological yield per plant $(-0.0213)$. Pods per cluster also showed positive direct effect on seed yield per plant and the results are in agreement with Haritha and Reddy (2002), Singh et al., (2009), Khanpara et al., (2012), Patel et al., (2014) and Hemavathy et al., (2015).

\section{Pods per plant}

At genotypic level, this trait showed significant positive association $\left(0.3178^{* *}\right)$ and positive direct effect (0.0934) on seed yield per plant. It also exhibited positive indirect effects via., days to maturity (0.2255), days to shattering (0.0029), plant height (0.0006), primary branches per plant $(0.0012)$, clusters per plant (0.0632), seeds per pod (0.0036), pod length (0.0058), 100-seed weight (0.0023), biological yield per plant (0.2055), harvest index (0.1073), protein content (0.0308) and seed hardness (0.0020), while negative indirect effects via., days to $50 \%$ flowering (-0.2133), leaf length (-0.0107), leaf width (-0.0006), pods per cluster ($0.013)$, shelling \% (-0.0015) and calcium content (-0.0004).

This trait also showed significant positive association $\left(0.3084^{* *}\right)$ and positive direct effects (0.0211) on seed yield per plant at phenotypic level. It also showed positive indirect effects via., days to maturity (0.0195), days to $50 \%$ flowering (0.0121), plant height (0.0002), leaf width (0.0027), clusters per plant (0.0409), pods per cluster $(0.0007)$, seeds per pod (0.0065), pod length (0.002), 100-seed weight (0.0038), shelling \% (0.0070), biological yield per plant (0.1650), harvest index (0.0974), protein content (0.0079), calcium content (0.0003) and seed hardness (0.0007), while negative indirect effects via., days to shattering (-0.0164), primary branches per plant $(-0.0038)$ and leaf length (-0.0169). Pods per plant showed positive direct effect on seed yield and the results are in agreement with Raturi et al., (2015), Muralidhara et al., (2015), Hemavathy et al., (2015), Dhoot et al., (2017), Kate et al., (2017) and Divyaramakrishnan et al., (2018).

\section{Seeds per pod}

This character showed non-significant positive association (0.1008) and positive direct effects (0.0507) on seed yield per plant at genotypic level. It also exhibited positive indirect effects via., days to $50 \%$ flowering (0.1106), primary branches per plant $(0.0010)$, clusters per plant (0.0119), pods per cluster (0.0051), pod length (0.0241), 100-seed weight (0.0002), biological yield per plant (0.0321), calcium content (0.0041), while negative indirect effects via., days to maturity $(-0.0832)$, days to shattering $(-0.0006)$, plant height $(-0.0074)$, leaf length $(-0.0027)$, leaf width $(-0.0007)$, pods per plant $(-0.0066)$, shelling \% (-0.0017), harvest index (-0.0097), protein content $(-0.0247)$ and seed hardness (0.0019 ) at genotypic level. This trait also showed non-significant positive association (0.1040) and positive direct effects (0.0864) on seed yield per plant at phenotypic level.

It also showed positive indirect effects via., days to shattering (0.0028), leaf width (0.0025), clusters per plant (0.0077), pod length (0.0087), 100-seed weight (0.0004), shelling \% (0.0072) and biological yield per plant (0.0270), while negative indirect effects via., days to $50 \%$ flowering (-0.0079), days to maturity (-0.0065), plant height (-0.0020), primary branches per plant $(-0.0034)$, leaf length (-0.0066), pods per cluster (-0.0003), pods per plant (-0.0016), harvest index($0.0014)$, protein content $(-0.0058)$, calcium content (-0.0028) and seed hardness (0.0007). For seeds per pod same results were 
showed by Srivastava and Singh (2012), Ahmad et al., (2013), Patel et al., (2014), Neem et al., (2015), Kumar and Katiyar (2015), Dhoot et al., (2017), Kritika and Yadav (2017) and Keerthiga et al., (2018).

\section{Pod length}

At genotypic level, the trait pod length showed significant positive association $\left(0.3049^{* *}\right)$ and positive direct effects $(0.0788)$ on seed yield per plant. It also exhibited positive indirect effects via., days to maturity (0.3282), days to shattering (0.0017), primary branches per plant (0.0004), pods per cluster $(0.0055)$, seeds per pod $(0.0155), 100$-seed weight (0.0058), biological yield per plant (0.1562), harvest index (0.0681) and protein content (0.0085), while negative indirect effects via., days to $50 \%$ flowering (-0.3148), plant height (-0.0199), leaf length (-0.0112), leaf width $(-0.0020)$, clusters per plant $(-$ $0.0011)$, pods per plant $(-0.0068)$, shelling \% $(-0.0044)$, calcium content $(-0.0023)$ and seed hardness $(-0.0012)$.

This trait showed significant positive association $\left(0.2537^{* * *}\right)$ and positive direct effects (0.0347) on seed yield per plant at phenotypic level. It also exhibited positive indirect effects via., days to maturity (0.0274), pods per cluster $(0.0055)$, seeds per pod (0.0218), 100-seed weight (0.0091), biological yield per plant $(0.1145)$, harvest index (0.0466), protein content (0.0014), shelling \% (0.0155), leaf width (0.0048) and days to $50 \%$ flowering $(0.0155)$. It also showed negative indirect effects via., days to shattering (-0.0154), plant height (-0.0042), primary branches per plant $(-0.0006)$, leaf length (-0.016), clusters per plant (-0.0009), pods per cluster $(-0.0002)$, pods per plant ($0.0012)$ and seed hardness (-0.0002). Arshad et al., (2009), Nand and Anuradha (2013) and Alom et al., (2014) showed same results for pod length.

\section{0-seed weight}

At genotypic level, this trait showed significant positive association $\left(0.4248^{* * *}\right)$ and positive direct effects $(0.0115)$ on seed yield per plant. It also showed positive indirect effects via., days to maturity (0.4000), days to shattering (0.0043), primary branches per plant (0.0009), clusters per plant (0.0240), seeds per pod (0.0007), pod length (0.0396), biological yield per plant (0.3001), harvest index (0.1011), protein content (0.0143), calcium content $(0.0025)$ and seed hardness (0.0011), while negative indirect effects via., days to $50 \%$ flowering (-0.4349), plant height $(-0.0076)$, leaf length $(-0.0075)$, leaf width $(-$ $0.0015)$, pods per cluster $(-0.0008)$, pods per plant (-0.0183) and shelling \% (-0.0048). The trait showed significant positive association $\left(0.3890^{* * *}\right)$ and positive direct effect $(0.0231)$ on seed yield per plant at phenotypic level. It also exhibited positive indirect effects via., days to $50 \%$ flowering (0.0227), days to maturity (0.0300), leaf width (0.0057), clusters per plant (0.0136), seeds per pod (0.0015), pod length (0.0136), shelling\% (0.0215), biological yield per plant (0.2097), harvest index(0.0854), protein content (0.0016) and seed hardness (0.0004), while negative indirect effects via., days to shattering (-0.0205), plant height (-0.0018), primary branches per plant $(-0.0028)$, leaf length (-0.0099) and calcium content ($0.0015)$. From the experimental findings, it was also observed that 100-seed weight had direct positive effect on seed yield per plant which has been confirmed earlier by Reddy et al., (2011), Hemavathy et al., (2015), Das and Barua (2015), Kumar and Katiyar (2015), Dhoot et al., (2017) and Keerthiga et al., (2018).

\section{Shelling \%}

At genotypic level, this character showed significant positive association $\left(0.5165^{* *}\right)$ and 
negative direct effects $(-0.0185)$ on seed yield per plant. It also exhibits positive indirect effects via., days to maturity (0.0214), days to shattering (0.0002), primary branches per plant (0.0001), leaf length (0.0020), leaf width (0.0002), clusters per plant (0.0218), seeds per pod (0.0046), pod length (0.0189), 100 -seed weight (0.0030), biological yield per plant (0.3256), harvest index $(0.1340)$, protein content (0.0217) and calcium content (0.0087), while negative indirect effects via., days to $50 \%$ flowering (-0.0080), plant height $(-0.0033)$, pods per cluster $(-0.0078)$, pods per plant (-0.0078) and seed hardness (-0.0002). The trait also showed significant positive association $\left(0.4929^{* *}\right)$ and positive direct effect (0.0851) on seed yield per plant at phenotypic level. It also exhibited positive indirect effects via., days to maturity (0.0023), days to shattering (0.0001), leaf length (0.0045), clusters per plant (0.0129), pods per cluster $(0.0003)$, seeds per pod (0.0073), pod length (0.0063), 100-seed weight (0.0058), biological yield per plant (0.2536), harvest index (0.1194), protein content (0.0042) and seed hardness (0.0001). It has showed negative indirect effects at phenotypic level via., plant height (-0.0008), primary branches per plant $(-0.0003)$, leaf weight $(-0.0006)$, pods per plant $(-0.0017)$ and calcium content (-0.0055).

\section{Biological yield per plant}

At genotypic level, this character showed significant positive association $\left(0.8113^{* *}\right)$ and positive direct effects $(0.7885)$ on seed yield per plant. It also exhibited positive indirect effects via., days to maturity (0.2789), days to shattering (0.0032), primary branches per plant (0.0009), leaf length (0.0062), leaf width (0.0001), clusters per plant (0.0359), pods per plant (0.0010), seeds per pod (0.0021), pod length (0.0156), 100-seed weight (0.0044), protein content (0.0143), calcium content $(0.0047)$, while negative indirect effects via., days to $50 \%$ flowering ($0.2970)$, plant height $(-0.0001)$, pods per plant $(-0.0244)$, shelling \% (-0.0076), harvest index $(-0.0130)$ and seed hardness $(-0.0023)$.

At phenotypic level, it showed significant positive association $\left(0.7497^{* *}\right)$ and positive direct effect $(0.6818)$ on seed yield per plant. It also exhibited positive indirect effects via., days to $50 \%$ flowering (0.0169), days to maturity (0.0222), leaf length (0.0090), clusters per plant $(0.0224)$, seeds per pod (0.0034), pod length (0.0058), 100-seed weight (0.0071), shelling \% (0.0317), protein content (0.0035), while negative indirect effects via., days to shattering (-0.0199), primary branches per plant $(-0.0029)$, leaf width (-0.0014), pods per cluster $(-0.0001)$, pods per plant (-0.0051), harvest index ($0.0203)$, calcium content (-0.0031) and seed hardness (-0.0008). Biological yield per plant had highest effect on seed yield per plant which was earlier reported by Mallikarjuna et al., (2006), Pandey et al., (2007), Singh et al., (2009), Gadakh et al., (2013) and Kritika and Yadav (2017).

\section{Harvest index}

At genotypic level, it showed significant positive association $\left(0.5009^{* * *}\right)$ and positive direct effect $(0.4867)$ on seed yield per plant. It also expressed positive indirect effects via., days to maturity $(0.2195)$, days to shattering $(0.0021)$, primary branches per plant $(0.0005)$, clusters per plant (0.0108), pod length (0.0110), 100-seed weight $(0.0024)$, protein content (0.0264) and seed hardness (0.0043), while negative indirect effects via., days to $50 \%$ flowering (-0.1872), plant height ($0.0085)$, leaf length $(-0.0041)$, leaf width ($0.0009)$, pods per cluster $(-0.0121)$, pods per plant (-0.0206), seeds per pod (-0.0010), shelling \% (-0.0051), biological yield per plant (-0.0211) and calcium content (-0.0022). At phenotypic level, this trait showed 
significant positive association $\left(0.4809^{* *}\right)$ and positive indirect effects $(0.4623)$ on seed yield per plant.

It also exhibited positive indirect effects via., days to $50 \%$ flowering (0.0098), days to maturity (0.0150), leaf width (0.0034), clusters per plant $(0.0063)$, pods per cluster (0.0006), pod length $(0.0035), 100$-seed weight $(0.0043)$, shelling \% (0.0220), protein content (0.0063), calcium content (0.0015) and seed hardness (0.0015), while negative indirect effects via., days to shattering ($0.0104)$, plant height $(-0.0022)$, primary branches per plant (-0.0015), leaf length ($0.0068)$, pods per plant $(-0.0044)$, seeds per pod (-0.0003), biological yield per plant (0.0300). Harvest index also showed positive direct effect which is as per the findings of Prasanna et al., (2013), Gadakh et al., (2013), Dhoot et al., (2017) and Kritika and Yadav (2017).

\section{Protein content}

At genotypic level, this trait showed significant negative association $\left(-0.2237^{* *}\right)$ and negative direct effects $(-0.1387)$ on seed yield per plant. It also exhibited positive indirect effects via., days to $50 \%$ flowering (0.2388), leaf length (0.0024), leaf width (0.0003), pods per cluster (0.0009), pods per plant (0.0207), seeds per pod (0.0090) and shelling \% (0.0029), while negative indirect effects via., days to maturity $(-0.1615)$, pod length (-0.0048), 100-seed weight (-0.0012), biological yield per plant $(-0.0810)$, harvest index (-0.0927), calcium content (-0.0019) and seed hardness (-0.0019). It showed significant negative association $\left(-0.1909^{* *}\right)$ and negative direct effect $(-0.0375)$ on seed yield per plant at phenotypic level. It also exhibited positive indirect effects via., days to shattering (0.0086), primary branches per plant (0.0004), leaf length (0.0044), pods per plant (0.0044), seeds per pod $(0.0133)$ and calcium content (0.0012), while negative indirect effects via., days to $50 \%$ flowering ($0.0130)$, days to maturity $(-0.0123)$, plant height $(-0.0006)$, leaf width (-0.0004), clusters per plant (-0.0062), pod length (-0.0013), 100 -seed weight $(-0.0010)$, shelling \% ($0.0094)$, biological yield per plant (-0.0631), harvest index (-0.0777) and seed hardness (0.0007). Wani et al., (2007) for seed protein.

\section{Calcium content}

At genotypic level, this trait showed nonsignificant negative association (-0.1378) and negative direct effect $(-0.0223)$ on seed yield per plant. It also exhibits positive indirect effects via., days to $50 \%$ flowering (0.0137), days to maturity (0.0256), pod length (0.0082), shelling \% (0.0072) and harvest index (0.0476), while it showed negative indirect effects via., days to shattering ($0.0004)$, plant height $(-0.0056)$, primary branches per plant $(-0.0003)$, leaf length ($0.0058)$, leaf width $(-0.0005)$, clusters per plant (-0.0109), pods per cluster $(-0.0022)$, pods per plant $(-0.0016)$, seeds per pod (0.0094), 100-seed weight (-0.0013), biological yield per plant $(-0.1659)$, protein content (-0.0119) and seed hardness (0.0020). At phenotypic level, this trait showed non-significant negative association (-0.1344) and positive direct effect $(0.0157)$ on seed yield per plant.

It also exhibited positive indirect effects via., days to maturity (0.0033), days to shattering $(0.0022)$, primary branches per plant $(0.0012)$, leaf width (0.0017), pods per cluster (0.0001), pod length (0.0029), harvest index (0.0433), while negative indirect effects via., days to $50 \%$ flowering (-0.0007), plant height ($0.0015)$, leaf length $(-0.0090)$, clusters per plant (-0.0072), pods per plant (-0.0004), seeds per pod $(-0.0153), 100$-seed weight ($0.0022)$, shelling \% (-0.1350), protein content $(-0.0029)$ and seed hardness $(-0.0007)$. 


\section{Seed hardness}

At genotypic level, this trait showed nonsignificant negative association $(-0.0086)$ and positive direct effect $(0.0212)$ on seed yield per plant. It also exhibited positive indirect effects via., days to 50\% flowering (0.1313), plant height (0.0063), primary branches per plant (0.0003), leaf width (0.0001), 100-seed weight (0.0006), shelling \% (0.0002), while negative indirect effects via., days to maturity $(-0.1506)$, days to shattering (-0.0016), leaf length (-0.0072), clusters per plant (-0.0089), pods per cluster $(-0.0099)$, pods per plant $(-$ $0.0087)$, seeds per pod (-0.0046), pod length $(-0.0044)$ and biological yield per plant ($0.0855)$. It showed non-significant negative association (-0.0010) and negative direct effects (-0.0010) on seed yield per plant at phenotypic level. It also showed positive indirect effects via., days to shattering (0.0107), plant height $(0.0017)$, clusters per plant (0.0005), pod length (0.0013), 100-seed weight (0.0009), biological yield per plant (0.0937), harvest index (0.0034) and calcium content (0.0076), while negative indirect effects via., days to $50 \%$ flowering (-0.0084), days to maturity $(-0.0145)$, primary branches per plant $(-0.0098)$, leaf length $(-0.0002)$, leaf width $(-0.0064)$, pods per cluster $(-0.0018)$, pods per plant $(-0.0078)$, seeds per pod ($0.0009)$, shelling \% (-0.0685) and protein content (-0.0014).

In present study, phenotypic and genotypic correlation of seed yield was positive and significant with days to $50 \%$ flowering, days to maturity, primary branches per plant, clusters per plant, pods per plant, pod length, 100 -seed weight, shelling \%, biological yield per plant and harvest index. This suggests while selecting for improvement in seed yield these characters can be kept in mind provided the character should show high variability, which is basis for selection. However, seed yield per plant showed significant and negative association with protein content in both genotypic and phenotypic correlation studies. Hence, these traits also can be emphasized while selecting for improvement of seed yield.

\section{References}

Arshad, M., Aslam, M. and Irshad, M. 2009. Genetic variability and character association among morphological traits of mung bean, Vigna radiata (L.) Wilczek genotypes. J Agric Res. 47(2): 121-126.

Bastian, L. D., Das, V., Kandasamy, G. and Sakila, M. 2001. Path analysis in cowpea (Vigna unguiculata (L.) Walp.). Madras Agric J. 88: 526-27.

Canci, H. and Toker, C. 2014. Yield components in mung bean [Vigna radiata (L.) Wilczek]. Turkish Journal of Field Crops. 19(2): 258-261.

Das, R. T. and Barua, P. K. 2015. Association studies for yield and its components in green gram. International Journal of Agriculture, Environment and Biotechnology. 8(3): 561-565.

Dewey, D. R and Lu, K. H. 1959. A correlation and path coefficient analysis of components of crested wheat grass seed production. Agronomy Journal. 51(9): 515-518.

Dhoot, R., Modha, K. G., Kumar, D., Dhoot, M. 2017. Correlations and path analysis Studies on yield and its components in mung bean (Vigna radiata (L.) Wilczek). International Journal of Current Microbiology and Applied Sciences. 6(5): 370- 378.

Divyaramakrishnan, C. K., Savithramma, D. L. and Vijayabharathi, A. 2018. Studies on genetic variability, correlation and path analysis for yield and yield related traits in green gram [Vigna radiata (L.) Wilczek]. International Journal of 
Current Microbiology and Applied Sciences. 7(3): 2319-7706.

Dixit, H. K., Singh, B. B. and Dua, R. P. 2002. Genetic variation in mungbean. Indian J Pulses Res. 15: 122-127.

Falconer, D. S. 1964. An Introduction to Quantitative Genetics. $2^{\text {nd }}$ ed. Oliver and Boyd Publishing Co. Pvt. Ltd. Edinburgh. 312-324.

Ghimire, S., Khanal, A., Kohar, G. R., Acharya, B., Basnet, A., Kandel, P., Subedi, B., Shrestha, J. and Dhaka, K. 2017. Variability and path coefficient analysis for yield attributing traits of mung bean (Vigna radiata L.). Azarian J. Agric. 5(1): 7- 11.

Gul, R., Khan, M., Mairaj, G., Ali, S., Farhatullah and Ikramullah. 2008. Correlation study on morphological and yield parameters of mung bean (Vigna radiata). Sarhad J Agric. 24: 43-45.

Hemavathy, A. T., Shunmugavalli, N. and Anand, G. 2015. Genetic variability, correlation and path co-efficient studies on yield and its components in mung bean [Vigna radiata $(\mathrm{L}$.) Wilczek]. Legume Research. 38(4): 442-446.

Kate, A. M., Dahat, D. V. and Chavan, B. H. 2017. Genetic variability, heritability, correlation and path analysis studies in green gram (Vigna radiata (L.) Wilczek). International Journal of Development Research. 7(11): 1670416707.

Keerthiga, S., Sen, S., Pandya, H. R. and Modha, K. G. 2018. Correlation and path analysis in $\mathrm{F}_{4}$ progenies of green gram [Vigna radiata (L.) Wilczek] for seed yield and its attributes. International Journal of Current Microbiology and Applied Sciences. 7(1): 2319-7706.

Khan, M. D., Khalil, I. H., Khan, M. A. and Ikramullah. 2004. Genetic divergence and association for yield and related traits in mung bean. Sarhad J. Agric. 20: 555-61.

Kritika and Yadav, R. 2017. Correlation and path coefficients analysis for seed yield and micronutrients in mung bean (Vigna radiata (L.) Wilczek). Int. J Pure App. Biosci. 5(1):908-917.

Kumar, R., Ravi Kant and Ojha, C. B. 2004. Character association and cause effect analysis for spring season genotypes of mung (Vigna radiata (L.) Wilczek). Legume Research. 27(1): 32-36.

Kumar, S. and Katiyar, M. 2015. Genetic variability, heritability, expected genetic advance and character association in mung bean (Vigna radiata L. Wilczek). International Journal of Advanced Research. 3(5): 1371-1375.

Lukman Hakim. 2008. Variability and correlation of agronomic characters of mung bean germplasm and their utilization for variety improvement program. Indonesian Journal of Agricultural Science. 9(1): 24-28.

Madrap, I. A., Dhuppe, M. V., Jahagirdar, J. E. and Hingole, D. G. 2003. Correlation and path analysis in mung bean. Proc Pulses for Crop Diversification and Natural Resource Management. Pp. 8487. Indian Institute of Pulse Research, Kanpur, India.

Muralidhara, Y. S., Kumar, L., Uday, B. M. G. and Shanthala, J. 2015. Studies on genetic variability, correlation and path analysis of seed yield and related traits in green gram [Vigna radiata $\mathrm{L}$. Wilczek]. International Journal of Agricultural Science and Research. 5(3):125-132.

Patel, S. R., Patel, K. K. and Parmar, H. K. 2014. Genetic variability, correlation and path analysis for seed yield and its components in green gram [Vigna radiata (L.) Wilczek]. The Bioscan. 9(4): 1847-1852. 
Raturi, A., Singh, S. K., Sharma, V. and Pathak, R. 2015. Genetic variability, heritability, genetic advance and path analysis in mung bean [Vigna radiata (L.) Wilczek]. Legume Research. 38(2): 157-163.

Reddy, D. K. R., Venkateswarlu, O., Obaiah, M. C. and Siva, J. G. L. 2011. Studies on genetic variability, character association and path co-efficient analysis in green gram [Vigna radiata (L.) Wilczek]. Legume Res. 34(3): 202-206.

Rozina, G., Khan, H. G., Farhatullah, S. and Ikramullah. 2008. Correlation study on morphological and yield parameters of mung bean (Vigna radiata). Sarhad Journal Agriculture. 24(1): 37-42.

Saleem, B., Ilyas, F., Ali, S., Qureshi, M. J. and Malik, I. A. 1998. Studies on chemical analysis of mung bean. (Vigna radiata (L.) Wilczek). Pakistan Journal. Bio. Sci., 1(2): 120-130.

Singh, T., Sharma, A., and Alie, F. A. 2009. Morpho-physiological traits as selection criteria for yield improvement in mung bean (Vigna radiata (L.) Wilczek). Legume Research. 32(1): 36-40.

Srivastava, R. L. and Singh, G. 2012. Genetic variability, correlation and path analysis in mung bean (Vigna radiata (L.) Wilczek). Indian J. L. Sci. 2: 61-65.

Tejbir, S., Sharma, A. and Alie, F. A. 2009. Morpho-physiological traits as selection criteria for yield improvement in mung bean [Vigna radiata $(\mathrm{L}$. Wilczek]. Legume Research. 32(1): 3640.

Venkatesan, M., Prakash, M. and Ganesan, J. 2003. Correlation and path analysis in cowpea (Vigna unguiculata (L.) Walp.). Legume Res. 26: 105-08.

Wani, B.A., Marker, S. and Lavanya, G. R. 2007. Genetic variability, correlation and path analysis in green gram. Journal of Mahararastra Agricultural University. 32(2): 216-219.

Wright, S. 1921. Correlation and causation. Journal of Agricultural Research. 20: $557-585$.

\section{How to cite this article:}

Jadhav, R.A., S.P. Mehtre and Patil, D.K. 2019. Correlation and Path Analysis Studies on Yield and its Components in Mung Bean [Vigna radiata (L.) Wilczek]. Int.J.Curr.Microbiol.App.Sci. 8(07): 699-720. doi: https://doi.org/10.20546/ijcmas.2019.807.086 